\title{
Amino Acid Conjugates of Aminothiazole and Aminopyridine as Potential Anticancer Agents: Synthesis, Molecular Docking and in vitro Evaluation
}

This article was published in the following Dove Press journal:

Drug Design, Development and Therapy

\author{
Shagufta $\mathrm{Naz}^{1,2}$ \\ Fawad Ali Shah (1D) \\ Humaira Nadeem' \\ Sadia Sarwar (ID) \\ Zhen $\operatorname{Tan}^{3}$ \\ Muhammad Imran (D) \\ Tahir Ali (iD ${ }^{2}$ \\ Jing Bo $\mathrm{Li}^{2}$ \\ Shupeng $\mathrm{Li}^{4}$ \\ 'Riphah Institute of Pharmaceutical \\ Sciences, Riphah International University, \\ Islamabad, 44000, Pakistan; ${ }^{2}$ Shenzhen \\ University Clinical Research Center for \\ Neurological Diseases, Health \\ Management Center, Shenzhen \\ University General Hospital, Shenzhen \\ University Clinical Medical Academy, \\ Shenzhen University, Shenzhen, People's \\ Republic of China; ${ }^{3}$ Hospital of Chengdu \\ University of Traditional Chinese \\ Medicine, Chengdu, People's Republic of \\ China; ${ }^{4}$ State Key Laboratory of \\ Oncogenomics, School of Chemical \\ Biology and Biotechnology, Shenzhen \\ Graduate School, Peking University, \\ Shenzhen, People's Republic of China
}

Correspondence: Humaira Nadeem Riphah Institute of Pharmaceutical

Sciences, Riphah International University, G-7/4, 7th Avenue, Islamabad, 44000,

Pakistan

Tel +92 51-2891835

Fax +92 5I-8350I80

Email humaira.nadeem@riphah.edu.pk

Shupeng Li

State Key Laboratory of Oncogenomics,

School of Chemical Biology and

Biotechnology, Shenzhen Graduate

School, Peking University, Shenzhen

People's Republic of China

Email lisp@pku.edu.cn
Purpose: The development of resistance to available anticancer drugs is increasingly becoming a major challenge and new chemical entities could be unveiled to compensate this therapeutic failure. The current study demonstrated the synthesis of 2-aminothiazole [S3 (a-d) and S5(a-d)] and 2-aminopyridine [S4(a-d) and S6(a-d)] derivatives that can target multiple cellular networks implicated in cancer development.

Methods: Biological assays were performed to investigate the antioxidant and anticancer potential of synthesized compounds. Redox imbalance and oxidative stress are hallmarks of cancer, therefore, synthesized compounds were preliminarily screened for their antioxidant activity using DPPH assay, and further five derivatives S3b, S3c, S4c, S5b, and S6c, with significant antioxidant potential, were selected for investigation of in vitro anticancer potential. The cytotoxic activities were evaluated against the parent (A2780) and cisplatin-resistant (A2780CISR) ovarian cancer cell lines. Further, Molecular docking studies of active compounds were performed to determine binding affinities.

Results: Results revealed that S3c, S5b, and S6c displayed promising inhibition in cisplatinresistant cell lines in comparison to parent cells in terms of both resistance factor (RF) and $\mathrm{IC}_{50}$ values. Moreover, S3c proved to be most active compound in both parent and resistant cell lines with $\mathrm{IC}_{50}$ values $15.57 \mu \mathrm{M}$ and $11.52 \mu \mathrm{M}$ respectively. Our docking studies demonstrated that compounds S3c, S5b, and S6c exhibited significant binding affinity with multiple protein targets of the signaling cascade.

Conclusion: Anticancer activities of compounds S3c, S5b, and S6c in cisplatin-resistant cell lines suggested that these ligands may contribute as lead compounds for the development of new anticancer drugs.

Keywords: thiazole, pyridine, antioxidant activity, anticancer activity, molecular docking

\section{Introduction}

Ovarian cancer is considered an immense human health hazard and it is the fifth leading cause of death worldwide. A woman's risk of getting invasive ovarian cancer is about 1 in 71 during her lifetime. ${ }^{1}$ Currently, new drug development has focused on the identification of novel targets and/or for ovarian cancer and its inhibitors. ${ }^{2}$ Over the past decades, most of the chemotherapeutic substances were designed against a specific target in the signaling network. Although these strategies initially increased curative efficacies, but the chances of re-occurrence are prevailing due to the increased resistance rate. Resistance likely develops due to the "rewiring" of subnetworks, including pathway re-programming and cross-activation 
in response to external stimuli. ${ }^{3}$ Thus, single-target therapies using highly specific compounds would likely fail in cancer treatment unless the compounds can disrupt multiple targets in the network. To deal with this problem, drug strategies called "network medicine" has been recently emerged to combat chemotherapeutic drug resistance. ${ }^{3-5}$ Epidermal growth factor receptor (EGFR), vascular endothelial growth factor receptor (VEGFR), plateletderived growth factor receptor (PDGFR), protein kinase $\mathrm{B}$ (PKB/AKT), mitogen-activated protein kinases (MAPKs), anaplastic lymphoma kinase (ALK), cytotoxic T-lymphocyte associated antigen-4 (CTLA-4), phosphoinositide-3-Kinase (PI3K) are the key signaling networks involved in the development of various types of cancers and are inhibited by different anticancer drugs. ${ }^{4-8}$ Furthermore, cisplatin and other platinum compounds have been used for several types of human malignancies, however, their efficacy is often compromised by tumor resistance. ${ }^{9}$ Moreover, currently marketed non-metallic anticancer drugs are also associated with intolerable side effects. ${ }^{10}$ To overcome this, new suitable alternative chemical entities must be ruled out which could offer considerable safety and efficacy. ${ }^{11}$ Several mechanisms are involved in cisplatin resistance and result in severe limitations in clinical use. ${ }^{12-14}$

Heterocyclic compounds are of great interest in the field of synthetic organic chemistry and medicinal chemistry. ${ }^{15,16}$ Thiazoles and pyridine are heterocyclic compounds and have demonstrated beneficial biological activities including antidiabetic, antimicrobial, antiviral, anti-inflammatory, anticancer, anti-alzheimer, antihypertensive, antioxidant, and hepatoprotective activities. ${ }^{17-27}$ Anticancer activities of the thiazole scaffold are well established as multiple therapeutically active agents like bleomycin, vosaroxin, epothilones, and dasatinib belong to this class. ${ }^{28}$ The groove-binding anticancer agents' such as dactinomycin, netropsin, and thia-netropsin do also share thiazole moiety in their structure. ${ }^{29}$ Other 2-aminothiazole analogues such as giroline ${ }^{30}$ and cantharidin ${ }^{31}$ exhibited increased apoptotic activity compared to their parent compounds. Moreover, pyridine derivatives such as imatinib mesylate (Gleevec) and aminopyridine derivatives have also been used as potential anticancer agents. ${ }^{32-35}$ Furthermore, free radicals have a significant detrimental propensity for DNA, proteins, and cellular membranes, and thus could exacerbate the pathogenesis of several diseases including cancer. ${ }^{36,37}$ Thus, by decreasing free radical generation and oxidative stress, antioxidants could ameliorate DNA damage, and consequently mutagenesis. ${ }^{38}$ Several studies reported the antioxidant activities of 2-aminothiazole and 2-aminopyridine derivatives. ${ }^{39,40}$ There is a great tendency to conjugate amino acid residues with various bioactive heterocyclic compounds in the field of biomedical research. Various products reported by conjugation of amino acid residue with heterocyclic motif resulted in enhanced potency, solubility, cell permeability, selectivity, in vivo stability, and decreased toxicity of heterocycles. ${ }^{41}$ Derivatives of aminothiazole with amino acids possess pronounced antibacterial and antifungal activities. ${ }^{41,42}$ Keeping in view the above literature and safety profile of amino acid conjugates, we presented in this study synthesis of new derivatives of ethyl-2-aminothiazole-4-carboxylate and 2-aminopyridine to explore their antioxidant and anticancer potential. Moreover, molecular docking studies against selected proteins from the cell signaling pathway also provided insight into the possible mechanism of active compounds at the molecular level to elaborate the concept of network medicine.

\section{Materials and Methods \\ Chemistry \\ General Information}

All the starting materials were purchased from SigmaAldrich (St. Louis, MO, USA), Daejung (South Korea), and Alfa-Aesar (Germany). Digital Gallenkamp (Sanyo) apparatus was used to record the melting points of final compounds and was uncorrected. Proton NMR ( ${ }^{1} \mathrm{H}$ NMR) and carbon-13 ( $\left.{ }^{13} \mathrm{C} \mathrm{NMR}\right)$ spectra were measured on a Bruker AV400 spectrophotometer in $\mathrm{CD}_{3} \mathrm{OD}, \mathrm{CDCl}_{3}$, and $\mathrm{DMSO}_{-} \mathrm{d}_{6}$ at $400 \mathrm{MHz}$ using TMS (Tetramethyl silane) as an internal standard. Alpha Bruker FTIR spectrophotometer (ATR eco $\mathrm{ZnSe}, v_{\max }$ in $\mathrm{cm}^{-1}$ ) was used to record FTIR spectra and elemental analysis was conducted using a LECO-183 CHNS analyzer. All reactions were monitored by thin-layer chromatography (TLC). Merck silica gel HF-254 was used for column chromatographic purification of products using (pet.ether: ethyl acetate, 4:1) as eluent. All chemicals used were of high analytical grade (99\% HPLC).

\section{General Procedure for the Preparation of Ethyl-2-Aminothiazole-4-Carboxylate (3)}

A mixture of ethyl bromopyruvate $(0.05 \mathrm{~mol}, 9.75 \mathrm{~g})$ and thiourea $(0.10 \mathrm{~mol}, 7.61 \mathrm{~g})$ in absolute ethanol $(53 \mathrm{~mL})$ was refluxed for $24 \mathrm{~h}$. The completion of the reaction was 
checked by thin layer chromatography (TLC). After completion, the reaction mixture was allowed to cool to room temperature and concentrated in vacuo to half the original volume. The remaining ethanol solution was poured into water and made alkaline ( $\mathrm{pH} 10)$ with $2 \mathrm{~N} \mathrm{NaOH}$. Light brown solid precipitated immediately. The mixture was stirred for about $10 \mathrm{~min}$ and then the solid was removed by vacuum filtration and dried to give the desired product. $^{47}$

\section{Ethyl 2-Amino-I,3-Thiazole-4-Carboxylate (3)}

Light brown crystals; yield: $68 \%$; m.p. found: $176{ }^{\circ} \mathrm{C}$; m.p. reported: $175-180{ }^{\circ} \mathrm{C} ; \mathrm{R}_{\mathrm{f}}=0.18$ (pet.ether:ethyl acetate $3: 1)$; FTIR $\left(v_{\max } \mathrm{cm}^{-1}\right) 3290\left(\mathrm{NH}_{2}, \mathrm{str}\right), 1733(\mathrm{C}=\mathrm{O}$, ester, str.), $1615\left(\mathrm{C}=\mathrm{N}\right.$, str.), $1540\left(\mathrm{C}=\mathrm{C}\right.$, str.); ${ }^{1} \mathrm{H}$ NMR (400 MHz, $\left.\mathrm{CD}_{3} \mathrm{OD}\right) \delta$ ppm 7.4 (s, 1H, H-1), 5.8 (brs, 2H, $\mathrm{NH}_{2}$ ), 4.32 (q, J=7.2Hz, 2H, H-2), 1.35 (t, J=7.2Hz, 3H, $\mathrm{H}-3$ ); ${ }^{13} \mathrm{C}$ NMR (400 MHz, $\left.\mathrm{CD}_{3} \mathrm{OD}\right) \delta \mathrm{ppm} 167.8,161.5$, 148.2, 116.7, 61.3, 15.2; Anal. calcd for $\mathrm{C}_{6} \mathrm{H}_{8} \mathrm{O}_{2} \mathrm{~N}_{2} \mathrm{~S}$ (172.20): C, 41.86; N, 16.27; H, 4.68. Found: C, 41.79; N, 16.19; H, 4.61\%.

\section{General Procedure for the Preparation of $N$,} $\mathrm{N}$-Phthaloyl Protected Amino Acids (SIa-SId)

A mixture of respective amino acids $(0.03 \mathrm{~mol})$ and finely ground phthalic anhydride $(0.03 \mathrm{~mol}, 4.44 \mathrm{~g})$ was heated with stirring in an oil bath at $185-190^{\circ} \mathrm{C}$ for $30 \mathrm{~min}$. After cooling, the solid material was dissolved in hot methanol $(20 \mathrm{~mL})$ and filtered. The product was allowed to crystallize out slowly in appropriate solvent (methanol/water, 1:1). ${ }^{48}$

( I,3-Dioxo-I,3-Dihydro-2H-Isoindol-2-yl)acetic Acid (SIa) White crystals; yield: $90 \%$; m.p. found: $197{ }^{\circ} \mathrm{C}$; m.p. reported: $197-198{ }^{\circ} \mathrm{C} ; \mathrm{R}_{\mathrm{f}}=0.64$ (ethyl acetate: pet.ether 3:1); FTIR ( $\left.v_{\max } \mathrm{cm}^{-1}\right) 1716(\mathrm{C}=\mathrm{O}$, acid, str. $), 1632,1536$ ( $\mathrm{C}=\mathrm{O}$, phthaloyl amide, str.); ${ }^{1} \mathrm{H} \mathrm{NMR}(400 \mathrm{MHz}$, $\left.\mathrm{CD}_{3} \mathrm{OD}\right) \delta \mathrm{ppm}$ 7.91-7.79 (m, 4H, Ar-H), 4.52 (s, 2H,

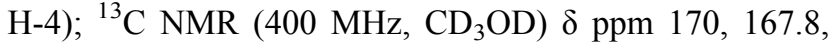
167.2, 134.7, 133.9, 131.9, 131.3, 122.6, 122.1, 38.7; Anal. calcd for $\mathrm{C}_{10} \mathrm{H}_{7} \mathrm{O}_{4} \mathrm{~N}$ (205.16): C, 58.53; N, 6.82; H, 3.41. Found: C, 58.51; N, 6.79; H, 3.38\%.

\section{2-(I,3-Dioxo-I,3-Dihydro-2H-Isoindol-2-yl)-}

3-Phenylpropanoic Acid (SIb)

White crystals; yield: 91\%; m.p. found: $177{ }^{\circ} \mathrm{C}$; m.p. reported: $178{ }^{\circ} \mathrm{C}$; Rf $=0.71$ (ethyl acetate: pet.ether $3: 1$ ); IR $\left(v_{\max } \mathrm{cm}^{-1}\right) 1714(\mathrm{C}=\mathrm{O}$, acid, str. $), 1636,1534(\mathrm{C}=\mathrm{O}$, phthaloyl amide, str.); ${ }^{1} \mathrm{H}$ NMR (400 MHz, $\left.\mathrm{CD}_{3} \mathrm{OD}\right) \delta \mathrm{ppm}$
7.90-7.77 (m, 8H, Ar-H), 5.41 (d, J=4.8Hz, 1H, H-4), 3.67 (m, 2H, H-5); ${ }^{13} \mathrm{C}$ NMR (400 MHz, $\left.\mathrm{CD}_{3} \mathrm{OD}\right) \delta$ ppm 176.5, $168.6,168.1,138.4,135.8,134.7,131.6,131.2,128.2$, 127.7, 125.5, 121.8, 121.1, 117.5, 117.2, 62.5, 35.3; Anal. calcd for $\mathrm{C}_{17} \mathrm{H}_{13} \mathrm{O}_{4} \mathrm{~N}$ (295.28): C, 69.15; N, 47.45; H, 4.41. Found: C, 69.05; N, 47.40; H, 4.38\%.

\section{2-(I,3-Dioxo- I,3-Dihydro-2H-Isoindol-2-yl)-}

4-Methylpentanoic Acid (SIc)

White crystals; yield: $83 \%$; m.p. found: $110{ }^{\circ} \mathrm{C}$; m.p. reported: $110{ }^{\circ} \mathrm{C} ; \mathrm{R}_{\mathrm{f}}=0.73$ (ethyl acetate: pet.ether $3: 1$ ); FTIR $\left(v_{\max } \mathrm{cm}^{-1}\right) 1718(\mathrm{C}=\mathrm{O}$, acid, str. $), 1633,1532(\mathrm{C}=\mathrm{O}$, phthaloyl amide, str.); ${ }^{1} \mathrm{H}$ NMR (400 MHz, $\left.\mathrm{CD}_{3} \mathrm{OD}\right) \delta \mathrm{ppm}$ 7.91 (d, 2H, J=8Hz, H-8, ', Ar-H), 7.78 (d, J=8Hz, 2H, H-9,9', Ar-H), 5.06 (dd, $\left.\mathrm{J}_{1}, \mathrm{~J}_{2}=4.8 \mathrm{~Hz}, 1 \mathrm{H}, \mathrm{H}-4\right), 2.40$ (m, 2H, H-5), 1.54 (m, 1H, H-6), 1.0 (d, J=6.4Hz, 6H, H-7a,7b); ${ }^{13} \mathrm{C}$ NMR (400 MHz, CD $\left.3 \mathrm{OD}\right) \delta$ ppm 180.3, 169.9, 169.3, 137.4, 136.3, 128.7, 128.4, 120.5, 120.1, 63.2, 37.5, 26.8, 20.9, 19.3; Anal. calcd for $\mathrm{C}_{14} \mathrm{H}_{15} \mathrm{O}_{4} \mathrm{~N}$ (261.27): C, 64.36; $\mathrm{N}$, 5.36; H, 5.75. Found: C, 64.30; N, 5.33; H, 5.71\%.

\section{2-(I,3-Dioxo-I,3-Dihydro-2H-Isoindol-2-yl)-}

3-Methylpentanoic Acid (SId)

White crystals; yield: $75 \%$; m.p. found: $112{ }^{\circ} \mathrm{C}$; m.p. reported: $110-112{ }^{\circ} \mathrm{C} ; \mathrm{R}_{\mathrm{f}}=0.8$ (ethyl acetate: pet.ether 3:1); FTIR ( $\left.v_{\max } \mathrm{cm}^{-1}\right) 1716(\mathrm{C}=\mathrm{O}$, acid, str. $), 1637,1530$ $\left(\mathrm{C}=\mathrm{O}\right.$, phthaloyl amide, str.); ${ }^{1} \mathrm{H}$ NMR (400 $\mathrm{MHz}$, $\left.\mathrm{CD}_{3} \mathrm{OD}\right) \delta$ ppm 7.87-7.79 (m, 4H, Ar-H), 4.60 (d, $\mathrm{J}=8 \mathrm{~Hz}, 1 \mathrm{H}, \mathrm{H}-4), 2.87$ (m, 1H, H-5), 1.57 (m, 2H, H-6), $1.11(\mathrm{~d}, \mathrm{~J}=8 \mathrm{~Hz}, 3 \mathrm{H}, \mathrm{H}-10), 0.91(\mathrm{t}, \mathrm{J}=8 \mathrm{~Hz}, 3 \mathrm{H}, \mathrm{H}-7)$; ${ }^{13} \mathrm{C}$ NMR (400 MHz, CD $\left.{ }_{3} \mathrm{OD}\right) \delta \mathrm{ppm} 174.6,165.7$, $165.3,135.5,134.4,127.8,126.3,118.7,118.4,65.3$, 36.8, 23.2, 16.6, 10.5; Anal. calcd for $\mathrm{C}_{14} \mathrm{H}_{15} \mathrm{O}_{4} \mathrm{~N}$ (261.27): C, 64.36; N, 5.36; H, 5.75. Found: C, 64.29; N, 5.30; H, 5.67\%.

\section{General Procedure for the Preparation of $N$, $\mathrm{N}$-Phthaloyl Amino Acid Chlorides (S2a-S2d)}

To a solution of respective $N, N$-phthaloyl amino acids $(0.005$ mol) in dichloromethane $(25 \mathrm{~mL})$, thionyl chloride $(0.015$ mol, $1.08 \mathrm{~mL}$ ) was added and refluxed for $8 \mathrm{~h}$. The progress of the reaction was monitored by TLC and after the completion, the excess of thionyl chloride was evaporated. The resulting product was used without further purification. ${ }^{49}$

\section{General Procedure for the Preparation of Amides (S3a-S3d and S4a-S4d)}

To a solution of respective $N, N$-phthaloyl amino acid chlorides $(0.01 \mathrm{~mol})$ in $20 \mathrm{~mL}$ of dichloromethane and $5 \mathrm{~mL}$ DMF 
(dimethylformamide), (0.01 mol, $2.06 \mathrm{~g})$ of DCC (dicyclohexyl carbodiimide) was added. The reaction mixture was stirred for $0.5 \mathrm{~h}$ followed by the dropwise addition of ethyl2-aminothiazole-4-carboxylate $(0.01 \mathrm{~mol}, 1.72 \mathrm{~g})$ or 2-aminopyridine $(0.01 \mathrm{~mol}, 0.94 \mathrm{~g})$ previously dissolved in $20 \mathrm{~mL}$ of dichloromethane. Further, the reaction mixture was stirred at $45^{\circ} \mathrm{C}$ for $2 \mathrm{~h}$ followed by overnight stirring at room temperature. The completion of the reaction was checked by TLC (pet.ether: ethyl acetate, 3:1). After completion, the mixture was allowed to cool, resulted in white dicyclohexylurea precipitates which were further removed by filtration. The excess of solvent was removed under reduced pressure and the corresponding product was dried and recrystallized from chloroform. ${ }^{50}$

Ethyl-2-\{[( I,3-Dioxo-I,3-Dihydro-2H-|soindol-2-yl)acetyl] amino\}-1,3-Thiazole-4-Carboxylate (S3a)

Brown solid; yield: $72 \%$; m.p. $171{ }^{\circ} \mathrm{C} ; \mathrm{R}_{\mathrm{f}}=0.37$ (pet.ether: ethyl acetate 3:1); FTIR $\left(v_{\max } \mathrm{cm}^{-1}\right) 3298(\mathrm{~N}-\mathrm{H}, \mathrm{str}), 1726$ $(\mathrm{C}=\mathrm{O}$, ester, str.), $1698(\mathrm{C}=\mathrm{O}$, amide, str.), 1631,1539 $(\mathrm{C}=\mathrm{O}$, phthaloyl amide, str.); ${ }^{1} \mathrm{H}$ NMR $\left(400 \mathrm{MHz}, \mathrm{CDCl}_{3}\right) \delta \mathrm{ppm} 9.25$ (brs, 1H, NH), 7.90-7.78 (m, 4H, aromatic), 7.28 (s, 1H, H-1), 4.52 (s, 2H, H-4), 3.74 (q, J=7.2Hz, 2H, H-2), 1.3 (t, J=7.2Hz, $3 \mathrm{H}, \mathrm{H}-3) ;{ }^{13} \mathrm{C}$ NMR (400 MHz, $\mathrm{CDCl}_{3}$ ) $\delta \mathrm{ppm} \mathrm{190.1,} \mathrm{170.4,}$ 167.5, 167.1, 158.2, 139.4, 133.6, 133.1, 130.4, 129.5, 123.8, 123.1, 113.5, 48.4, 39.6, 6.9; Anal. calcd for $\mathrm{C}_{16} \mathrm{H}_{13} \mathrm{O}_{5} \mathrm{~N}_{3} \mathrm{~S}$ (343.35): C, 53.48; N, 11.69; H, 3.62. Found: C, $52.70 ; \mathrm{N}, 10.45 ; \mathrm{H}, 3.10 \%$.

\section{Ethyl-2-\{[2-(I,3-Dioxo-I,3-Dihydro-2H-|soindol-2-yl)-} 3-Phenyl]amino\}-1,3-Thiazole-4-Carboxylate (S3b)

White crystals; yield: $88 \%$; m.p. $153^{\circ} \mathrm{C} ; \mathrm{R}_{\mathrm{f}}=0.29$ (pet.ether: ethyl acetate 3:1); FTIR $\left(v_{\max } \mathrm{cm}^{-1}\right) 3322(\mathrm{~N}-\mathrm{H}, \mathrm{str}), 1730$ ( $\mathrm{C}=\mathrm{O}$, ester, str.), 1695 ( $\mathrm{C}=\mathrm{O}$, amide, str.), 1662,1546 (C=O, phthaloyl amide, str.); ${ }^{1} \mathrm{H}$ NMR (400 MHz, $\left.\mathrm{CDCl}_{3}\right) \delta \mathrm{ppm}$ 10.11 (brs, $1 \mathrm{H}, \mathrm{NH}), 7.85-7.77$ (m, 8H, Ar-H), 7.17 (s, 1H, $\mathrm{H}-1), 5.39$ (d, J=4.8Hz, 1H, H-4), 4.42 (q, J=7.2Hz, 2H, H-2), 3.67 (m, 2H, H-5), 1.40 (t, J=7.2Hz, 3H, H-3); ${ }^{13} \mathrm{C} \mathrm{NMR}$ $\left(400 \mathrm{MHz}, \mathrm{CDCl}_{3}\right) \delta \mathrm{ppm} 191.2,170.5,167.7,167.3,156.4$, 138.2, 137.4, 133.8, 132.1, 131.4, 131.1, 130.2, 129.8, 129.1, 128.7, 128.2, 123.5, 123.3, 115.4, 58.2, 37.9, 35.2, 8.3; Anal. calcd for $\mathrm{C}_{23} \mathrm{H}_{19} \mathrm{O}_{5} \mathrm{~N}_{3} \mathrm{~S}$ (433.47): C, 61.46; N, 9.35; H, 4.23. Found: C, 60.39; N, 8.51; H, 3.95\%.

\section{Ethyl-2-\{[2-(I,3-Dioxo-I,3-Dihydro-2H-Isoindol-2-yl)-} 4-Methylpentanoyl]amino\}-1,3-Thiazole-4-Carboxylate (S3c) White crystals; yield: $86 \%$; m.p. $79{ }^{\circ} \mathrm{C} ; \mathrm{R}_{\mathrm{f}}=0.36$ (pet.ether: ethyl acetate 3:1); FTIR $\left(v_{\max } \mathrm{cm}^{-1}\right) 3359$ (N-H, str.), 1733
$(\mathrm{C}=\mathrm{O}$, ester, str.), 1696 ( $\mathrm{C}=\mathrm{O}$, amide, str.), 1650,1563 ( $\mathrm{C}=\mathrm{O}$, phthaloyl amide, str.); ${ }^{1} \mathrm{H}$ NMR $\left(400 \mathrm{MHz}, \mathrm{CDCl}_{3}\right) \delta \mathrm{ppm}$ 9.78 (brs, $1 \mathrm{H}, \mathrm{NH}), 7.91-7.83(\mathrm{~m}, 4 \mathrm{H}, \mathrm{Ar}-\mathrm{H}), 7.29$ (s, 1H, $\mathrm{H}-1), 5.19$ (dd, $\left.\mathrm{J}_{1}, \mathrm{~J}_{2}=4.8 \mathrm{~Hz}, 1 \mathrm{H}, \mathrm{H}-4\right), 4.42$ (q, J=7.2Hz, $2 \mathrm{H}$, $\mathrm{H}-2), 2.20$ (m, 1H, H-6), 1.60 (m, 2H, H-5), 1.41 (t, J=7.2Hz, $3 \mathrm{H}, \mathrm{H}-3), 1.0(\mathrm{~d}, \mathrm{~J}=6.4 \mathrm{~Hz}, 6 \mathrm{H}, \mathrm{H}-7 \mathrm{a}, 7 \mathrm{~b}) ;{ }^{13} \mathrm{C} \mathrm{NMR}(400$ $\left.\mathrm{MHz}, \mathrm{CDCl}_{3}\right) \delta$ ppm 189.4, 171.2, 168.5, 167.8, 157.1, 138.4, 134.4, 133.1, 132.4, 131.3, 124.6, 123.1, 117.5, 61.8, $36.9,33.5,26.2,23.4,22.5,7.4$; Anal. calcd for $\mathrm{C}_{20} \mathrm{H}_{21} \mathrm{O}_{5} \mathrm{~N}_{3} \mathrm{~S}$ (399.46): C, 57.83; N, 10.12; H, 5.06. Found: C, 56.45; N, 9.89; H, 4.82\%.

\section{Ethyl-2-\{[2-(I,3-Dioxo-I,3-Dihydro-2H-Isoindol-2-yl)-} 3-Methylpentanoy|]amino\}-1,3-Thiazole-4-Carboxylate (S3d) White crystals; yield: $78 \%$; m.p. $174^{\circ} \mathrm{C} ; \mathrm{R}_{\mathrm{f}}=0.34$ (pet.ether: ethyl acetate 3:1); FTIR $\left(v_{\max } \mathrm{cm}^{-1}\right) 3320(\mathrm{~N}-\mathrm{H}$, str.), 1728 $(\mathrm{C}=\mathrm{O}$, ester, str.), $1692(\mathrm{C}=\mathrm{O}$, amide, str.), 1642,1521 ( $\mathrm{C}=\mathrm{O}$, phthaloyl amide, str.); ${ }^{1} \mathrm{H}$ NMR (400 MHz, $\left.\mathrm{CDCl}_{3}\right) \delta \mathrm{ppm}$ 10.32 (brs, $1 \mathrm{H}, \mathrm{NH}), 7.87-7.79$ (m, 4H, Ar-H), 7.28 (s, 1H, $\mathrm{H}-1), 4.78$ (q, J=7.2Hz, 2H, H-2), 4.43 (d, J=8Hz, 1H, H-4), 2.87 (m, 1H, H-5), 1.57 (m, 2H, H-6), 1.42 (t, J=7.2Hz, 3H, $\mathrm{H}-3), 1.11$ (d, J=8Hz 3H, H-10), 0.91 (t, J=8Hz, 3H, H-7); ${ }^{13} \mathrm{C}$ NMR (400 MHz, $\left.\mathrm{CDCl}_{3}\right) \delta$ ppm 190.4, 171.5, 167.5, 167.1, 156.3, 139.4, 133.6, 133.1, 132.8, 131.7, 123.6, 123.1, 115.3, 58.5, 34.2, 31.5, 27.2, 19.2, 12.6, 6.8; Anal. calcd for $\mathrm{C}_{20} \mathrm{H}_{21} \mathrm{O}_{5} \mathrm{~N}_{3} \mathrm{~S}$ (399.46): C, 57.83; N, 10.12; H, 5.06. Found: C, $56.58 ; \mathrm{N}, 9.91 ; \mathrm{H}, 4.87 \%$.

\section{2-(I,3-Dioxo-I,3-Dihydro-2H-Isoindol-2-yl)-N-(Pyridin-}

\section{2-yl)acetamide (S4a)}

Off-white solid; yield: $64 \%$; m.p. $134{ }^{\circ} \mathrm{C} ; \mathrm{R}_{\mathrm{f}}=0.36$ (pet. ether:ethyl acetate 3:1); FTIR $\left(v_{\max } \mathrm{cm}^{-1}\right) 3296(\mathrm{~N}-\mathrm{H}$, str.), $1690 \quad(\mathrm{C}=\mathrm{O}$, amide, str.), 1647,1552 (C=O, phthaloyl amide, str.); ${ }^{1} \mathrm{H}$ NMR (400 MHz, $\mathrm{CDCl}_{3}$ ) $\delta$ ppm 9.11 (brs, $1 \mathrm{H}, \mathrm{NH}), 8.67$ (d, J=4.1 Hz, 1H, pyridine), 8.29 (d, $\mathrm{J}=7.9 \mathrm{~Hz}, 1 \mathrm{H}$, pyridine $), 7.83(\mathrm{t}, \mathrm{J}=7.8 \mathrm{~Hz}, 1 \mathrm{H}$, pyridine $)$, $7.70\left(\mathrm{dd}, \mathrm{J}_{1}, \mathrm{~J}_{2}=7.2 \mathrm{~Hz}, 1 \mathrm{H}\right.$, pyridine $), 7.24-7.04(\mathrm{~m}, 4 \mathrm{H}$, $\mathrm{Ar}-\mathrm{H}), 4.52$ (s, 2H, glycine); ${ }^{13} \mathrm{C} \mathrm{NMR}\left(400 \mathrm{MHz}, \mathrm{CDCl}_{3}\right)$ $\delta$ ppm 170.5, 168.1, 167.8, 155.4, 149.2, 138.4, 134.4, $133.2,131.8,130.4,124.8,122.3,119.2,114.5,43.7$; Anal. calcd for $\mathrm{C}_{16} \mathrm{H}_{13} \mathrm{O}_{5} \mathrm{~N}_{3} \mathrm{~S}$ (343.35): C, 53.48; N, 11.69; H, 3.62. Found: C, 53.42; N, 11.60 ; H, 3.59\%.

\section{2-(I,3-Dioxo-I,3-Dihydro-2H-Isoindol-2-yl)-3-Phenyl-} $\mathrm{N}$-(Pyridin-2-yl)propanamide (S4b)

White crystals; yield: $85 \%$; m.p. $174{ }^{\circ} \mathrm{C} ; \mathrm{R}_{\mathrm{f}}=0.27$ (pet. ether:ethyl acetate 3:1); FTIR $\left(v_{\max } \mathrm{cm}^{-1}\right) 3313(\mathrm{~N}-\mathrm{H}, \mathrm{str})$, 1698 (C=O, amide, str.), 1644,1523 ( $\mathrm{C}=\mathrm{O}$, phthaloyl 
amide, str.); ${ }^{1} \mathrm{H}$ NMR $\left(400 \mathrm{MHz}, \mathrm{CDCl}_{3}\right) \delta$ ppm 9.85 (brs, $1 \mathrm{H}, \mathrm{NH}), 8.63$ (d, J=4.3 Hz, 1H, pyridine), 8.30 (d, $\mathrm{J}=8 \mathrm{~Hz}, 1 \mathrm{H}$, pyridine), 7.81 ( $\mathrm{t}, \mathrm{J}=7.7 \mathrm{~Hz}, 1 \mathrm{H}$, pyridine), 7.70 (dd, $\mathrm{J}_{1}, \mathrm{~J}_{2}=7.1 \mathrm{~Hz}, 1 \mathrm{H}$, pyridine), 7.20-7.06 (m, 9H, Ar-H), 5.31 (d, J=4.8 Hz 1H, CH- $\alpha$-carbon), 3.69 (m, $2 \mathrm{H}, \mathrm{CH}_{2-}$ phenyl); ${ }^{13} \mathrm{C}$ NMR (400 MHz, $\left.\mathrm{CDCl}_{3}\right) \delta \mathrm{ppm} \mathrm{171.2,}$ $167.1,166.9,152.3,148.2,139.6,137.2,134.2,133.5$, $131.5,131.1,129.9,129.1,127.5,126.9,125.8,124.8$, 123.7, 118.2, 113.7, 62.6, 39.2; Anal. calcd for $\mathrm{C}_{23} \mathrm{H}_{19} \mathrm{O}_{5} \mathrm{~N}_{3} \mathrm{~S}$ (433.47): C, 61.46; N, 9.35; H, 4.23. Found: C, 61.39; N, 9.31; H, 4.20\%.

\section{2-(I,3-Dioxo-I,3-Dihydro-2H-|soindol-2-yl)-4-Methyl- $\mathrm{N}$-(Pyridin-2-yl)pentanamide (S4c)}

White crystals; yield: $60 \%$; m.p. $109^{\circ} \mathrm{C} ; \mathrm{R}_{\mathrm{f}}=0.325$ (pet. ether:ethyl acetate 3:1); FTIR $\left(v_{\max } \mathrm{cm}^{-1}\right) 3343(\mathrm{~N}-\mathrm{H}$, str.), 1697 ( $\mathrm{C}=\mathrm{O}$, amide, str.), 1635,1542 ( $\mathrm{C}=\mathrm{O}$, phthaloyl amide, str.); ${ }^{1} \mathrm{H}$ NMR (400 MHz, $\left.\mathrm{CDCl}_{3}\right) \delta$ ppm 9.68 (brs, $1 \mathrm{H}, \mathrm{NH}$ ), $8.65(\mathrm{~d}, \mathrm{~J}=4 \mathrm{~Hz}, 1 \mathrm{H}$, pyridine), $8.29(\mathrm{~d}, \mathrm{~J}=7.9 \mathrm{~Hz}, 1 \mathrm{H}$, pyridine), 7.92 (t, $\mathrm{J}=7.8 \mathrm{~Hz}, 1 \mathrm{H}$, pyridine), 7.79 (dd, $\mathrm{J}_{1} \mathrm{~J}_{2}=7 \mathrm{~Hz}$, 1H, pyridine), 7.28-7.06 (m, 4H, Ar- $\mathrm{H}), 5.11(\mathrm{t}, \mathrm{J}=5 \mathrm{~Hz}, 1 \mathrm{H}$, $\mathrm{CH}$ - $\alpha$-carbon), $2.01\left(\mathrm{~m}, 1 \mathrm{H},-\mathrm{CH}\left(\mathrm{CH}_{3}\right)_{2}\right.$-leucine), 1.56 (dd, $2 \mathrm{H},-\mathrm{CH}_{2}$-leucine $), 1.0\left(\mathrm{~d}, 6 \mathrm{H}, \mathrm{J}=6.4 \mathrm{~Hz},-\mathrm{CH}\left(\mathrm{CH}_{3}\right)_{2}\right.$-leucine); ${ }^{13} \mathrm{C}$ NMR (400 MHz, $\left.\mathrm{CDCl}_{3}\right) \delta$ ppm 171.6, 167.9, 167.1, 157.4, 148.4, 136.5, 134.8, 134.2, 132.9, 131.4, 125.8, 124.2, 118.4, 115.5, 69.5, 38.7, 30.4, 29.5, 23.5; Anal. calcd for $\mathrm{C}_{20} \mathrm{H}_{21} \mathrm{O}_{5} \mathrm{~N}_{3} \mathrm{~S}$ (399.46): C, 57.83; N, 10.12; $\mathrm{H}, 5.06$. Found: C, 57.80; N, 10.02; H, 5.02\%.

\section{2-(I,3-Dioxo-I,3-Dihydro-2H-|soindol-2-yl)-3-Methyl-} $\mathrm{N}-($ Pyridin-2-yl)pentanamide (S4d)

White crystals; yield: $54 \%$; m.p. $107^{\circ} \mathrm{C} ; \mathrm{R}_{\mathrm{f}}=0.35$ (pet.ether: ethyl acetate 3:1); FTIR $\left(v_{\max } \mathrm{cm}^{-1}\right) 3310$ (N-H, str.), 1695 ( $\mathrm{C}=\mathrm{O}$, amide, str.), 1640,1531 ( $\mathrm{C}=\mathrm{O}$, phthaloyl amide, str.); ${ }^{1} \mathrm{H}$ NMR (400 MHz, CDCl ${ }_{3}$ ) $\delta$ ppm 9.96 (brs,1H, NH), 9.49 (d, $\mathrm{J}=4 \mathrm{~Hz}, 1 \mathrm{H}$, pyridine), 8.32 (d, $\mathrm{J}=8 \mathrm{~Hz}, 1 \mathrm{H}$, pyridine), 8.20 (t, J=7.8Hz, $1 \mathrm{H}$, pyridine), 7.90 (dd, $\mathrm{J}_{1} \mathrm{~J}_{2}=7.2 \mathrm{~Hz}, 1 \mathrm{H}$, pyridine), 7.28-7.03 (m, 4H, Ar-H), $4.66(\mathrm{~d}, \mathrm{~J}=8 \mathrm{~Hz}, 1 \mathrm{H}, \alpha-$ carbon), $2.87\left(\mathrm{~m}, 1 \mathrm{H},-\mathrm{CHCH}_{3}\right.$-isoleucine), $1.57(\mathrm{~m}, 2 \mathrm{H}$, $-\mathrm{CH}_{2} \mathrm{CH}_{3}$-isoleucine), $1.11\left(\mathrm{~d}, \mathrm{~J}=8 \mathrm{~Hz}, 3 \mathrm{H}\right.$, $-\mathrm{CHCH}_{3}$-isoleucine), 0.91 ( $\mathrm{t}, \mathrm{J}=8 \mathrm{~Hz}, 3 \mathrm{H},-\mathrm{CH}_{2} \mathrm{CH}_{3}$-isoleucine); ${ }^{13} \mathrm{C} \mathrm{NMR}$ $\left(400 \mathrm{MHz}, \mathrm{CDCl}_{3}\right) \delta \mathrm{ppm} 170.4,168.5,167.9,154.3,149.2$, 137.6, 133.8, 132.2, 131.9, 131.1, 124.8, 123.2, 117.5, 116.2, 58.5, 32.7, 29.3, 17.6, 12.3; Anal. calcd for $\mathrm{C}_{20} \mathrm{H}_{21} \mathrm{O}_{5} \mathrm{~N}_{3} \mathrm{~S}$ (399.46): C, 57.83; N, 10.12; H, 5.06. Found: C, 57.78; N, 10.10; H, 5.01\%.

\section{General Procedure for N-Deprotection of Amino} Acids (S5a-S5d and S6a-S6d)

To a solution of respective phthalimides $(0.01 \mathrm{~mol})$ in boiling ethanol $(22 \mathrm{~mL})$, hydrazine hydrate $50 \%(0.01$ mol, $0.32 \mathrm{~g}$ ) was added. The completion of the reaction was checked by TLC. On cooling, the precipitates were collected, and recrystallized from ethanol. ${ }^{51}$

2-Amino-N-[4-(Hydrazinylcarbonyl)-I,3-Thiazol-2-yl]acetamide (S5a)

Brown solid; yield: $62 \%$; m.p. $195{ }^{\circ} \mathrm{C} ; \mathrm{R}_{\mathrm{f}}=0.37$ (pet. ether:ethyl acetate 3:1); FTIR $\left(v_{\max } \mathrm{cm}^{-1}\right) 3298$ (N-H, str.), 3276 (N-H, str.), 3268 ( $\mathrm{NH}_{2}$, str.), $3263\left(\mathrm{NH}_{2}\right.$, str.), 1698 $\left(\mathrm{C}=\mathrm{O}\right.$, amide, str.), $1646(\mathrm{C}=\mathrm{O}$, amide, str. $) ;{ }^{1} \mathrm{H}$ NMR (400 MHz, DMSO-d ${ }_{6}$ ) $\delta$ ppm 9.25 (brs, $\left.1 \mathrm{H}, \mathrm{NH}\right), 8.79$ (s, 1H, NHCO), 7.28 (s, 1H, H-1), 4.52 (s, 2H, H-4), 4.38 (s, 2H, $\left.\mathrm{NH}_{2} \mathrm{NH}\right), 4.25\left(\mathrm{~s}, 2 \mathrm{H}, \mathrm{NH}_{2}\right) ;{ }^{13} \mathrm{C}$ NMR $(400 \mathrm{MHz}$, DMSO-d $\left._{6}\right) \delta$ ppm 169.8, 162.4, 159.4, 150.4, 114.5, 45.2; Anal. calcd for $\mathrm{C}_{6} \mathrm{H}_{9} \mathrm{O}_{2} \mathrm{~N}_{5} \mathrm{~S}$ (215.23): C, 33.48; N, 32.54; H, 4.21. Found: C, 33.42; N, 32.40; H, 4.10\%.

\section{2-Amino-N-[4-(Hydrazinylcarbonyl)-I,3-Thiazol-2-yl]-}

\section{3-Phenylpropanamide (S5b)}

Light brown solid; yield: 88\%; m.p. $206{ }^{\circ} \mathrm{C} ; \mathrm{R}_{\mathrm{f}}=0.29$ (pet.ether:ethyl acetate 3:1); FTIR $\left(v_{\max } \mathrm{cm}^{-1}\right) 3299(\mathrm{~N}-\mathrm{H}$, str.), 3273 (N-H, str.), 3269 ( $\mathrm{NH}_{2}$, str.), $3261\left(\mathrm{NH}_{2}\right.$, str.), 1696 ( $\mathrm{C}=0$, amide, str.), 1646 ( $\mathrm{C}=\mathrm{O}$, amide, str.); ${ }^{1} \mathrm{H}$ NMR $\left(400 \mathrm{MHz}, \mathrm{DMSO}-\mathrm{d}_{6}\right) \delta \mathrm{ppm} 10.11$ (brs,1H, NH), 8.89 (s, 1H, NHCO), 7.85-7.77 (m, 5H, Ar-H), 7.17 (s, 1H, H-1), $5.39(\mathrm{~d}, \mathrm{~J}=4.8 \mathrm{~Hz}, 1 \mathrm{H}, \mathrm{H}-4), 4.39$ (s, $\left.2 \mathrm{H}, \mathrm{NH}_{2} \mathrm{NH}\right), 4.28$ (s, $\left.2 \mathrm{H}, \mathrm{NH}_{2}\right), 3.67(\mathrm{~m}, 2 \mathrm{H}, \mathrm{H}-5) ;{ }^{13} \mathrm{C}$ NMR $(400 \mathrm{MHz}$, DMSO-d $\left.\mathrm{d}_{6}\right) \delta \mathrm{ppm} 174.2,163.7,160.1,150.2,137.4$, $129.8,128.5,128.1,127.9,126.3,120.5,49.8,34.2$; Anal. calcd for $\mathrm{C}_{13} \mathrm{H}_{15} \mathrm{O}_{2} \mathrm{~N}_{5} \mathrm{~S}$ (305.35): C, 51.13; N, 22.94; H, 4.95. Found: C, 51.10; N, 22.89; H, 4.92\%.

\section{2-Amino-N-[4-(Hydrazinylcarbonyl)-I,3-Thiazol-2-yl]- 4-Methylpentanamide (S5c)}

Orange solid; yield: $70 \%$; m.p. $198{ }^{\circ} \mathrm{C}$; $\mathrm{R}_{\mathrm{f}}=0.36$ (pet. ether:ethyl acetate 3:1); FTIR $\left(v_{\max } \mathrm{cm}^{-1}\right) 3298$ (N-H, str.), 3275 (N-H, str.), $3266\left(\mathrm{NH}_{2}\right.$, str.), $3260\left(\mathrm{NH}_{2}\right.$, str.), 1695 ( $\mathrm{C}=0$, amide, str.), 1647 ( $\mathrm{C}=\mathrm{O}$, amide, str.); ${ }^{1} \mathrm{H}$ NMR (400 MHz, DMSO-d $\left.{ }_{6}\right) \delta$ ppm 9.78 (brs, $\left.1 \mathrm{H}, \mathrm{NH}\right), 8.77$ (s, 1H, $\mathrm{NHCO}), 7.29$ (s, 1H, H-1), 5.19 (dd, $\mathrm{J}_{1} \mathrm{~J}_{2}=4.8 \mathrm{~Hz}, 1 \mathrm{H}$, $\mathrm{H}-4), 4.38$ (s, $\left.2 \mathrm{H}, \mathrm{NH}_{2} \mathrm{NH}\right), 4.26$ (s, $\left.2 \mathrm{H}, \mathrm{NH}_{2}\right), 2.20(\mathrm{~m}$, $1 \mathrm{H}, \mathrm{H}-6), 1.60(\mathrm{~m}, 2 \mathrm{H}, \mathrm{H}-5), 1.0(\mathrm{~d}, \mathrm{~J}=6.4 \mathrm{~Hz}, 6 \mathrm{H}$, $\mathrm{H}-7 \mathrm{a}, 7 \mathrm{~b}) ;{ }^{13} \mathrm{C}$ NMR (400 MHz, DMSO-d 6 ) $\delta$ ppm 170.4, $161.5,158.2,147.8,114.5,53.1,45.1,20.4,19.2,17.3$; 
Anal. calcd for $\mathrm{C}_{10} \mathrm{H}_{17} \mathrm{O}_{2} \mathrm{~N}_{5} \mathrm{~S}$ (271.33): $\mathrm{C}, 44.26 ; \mathrm{N}$, 25.81; H, 6.31. Found: C, 44.21; N, 25.79; H, 6.28\%.

2-Amino-N-[4-(Hydrazinylcarbonyl)-I,3-Thiazol-2-yl]3-Methylpentanamide (S5d)

Brown solid; yield: 78\%; m.p. $161{ }^{\circ} \mathrm{C} ; \mathrm{R}_{\mathrm{f}}=0.34$ (pet.ether: ethyl acetate 3:1); FTIR ( $\left.v_{\max } \mathrm{cm}^{-1}\right) 3301$ (N-H, str.), 3278 (N-H, str.), $3265\left(\mathrm{NH}_{2}\right.$, str.), $3262\left(\mathrm{NH}_{2}\right.$, str.), $1692(\mathrm{C}=\mathrm{O}$, amide, str.), 1646 (C=O, amide, str.); ${ }^{1} \mathrm{H}$ NMR (400 MHz, DMSO-d $\left._{6}\right) \delta$ ppm 10.32 (brs,1H, NH), 8.79 (s, 1H, NHCO), 7.28 (s, 1H, H-1), 4.43 (d, J=8Hz, 1H, H-4), 4.37 (s, 2H, $\left.\mathrm{NH}_{2} \mathrm{NH}\right), 4.28$ (s, 2H, $\left.\mathrm{NH}_{2}\right), 2.87$ (m, 1H, H-5), 1.57 (m, 2H, H-6), 1.11 (d, J=8Hz 3H, H-10), 0.91 (t, J=8Hz, 3H, H-7); ${ }^{13} \mathrm{C}$ NMR (400 MHz, DMSO-d ${ }_{6}$ ) $\delta$ ppm 172.4, 164.4, 158.9, 146.1, 116.5, 58.5, 39.4, 24.8, 18.4, 10.1; Anal. calcd for $\mathrm{C}_{10} \mathrm{H}_{17} \mathrm{O}_{2} \mathrm{~N}_{5} \mathrm{~S}$ (271.33): C, 44.26; N, 25.81; H, 6.31. Found: C, 44.20; N, 25.80; H, 6.29\%.

\section{2-Amino-N-(Pyridin-2-yl)acetamide (S6a)}

Off-white solid; yield: 65\%; m.p. $204{ }^{\circ} \mathrm{C}$; $\mathrm{R}_{\mathrm{f}}=0.36$ (pet. ether:ethyl acetate $3: 1)$; FTIR $\left(v_{\max } \mathrm{cm}^{-1}\right) 3296$ (N-H, str.), $3263\left(\mathrm{NH}_{2}\right.$, str. $), 1690$ (C=O, amide, str.); ${ }^{1} \mathrm{H}$ NMR (400 MHz, DMSO-d 6 ) $\delta$ ppm 9.11 (brs, $1 \mathrm{H}, \mathrm{NH}$ ), 8.67 (d, J=4.1 $\left.\mathrm{Hz}, 1 \mathrm{H}, \mathrm{H}-3^{\prime}\right), 8.29$ (d, J=7.9Hz, 1H, H-6'), 7.83 (t, $\left.\mathrm{J}=7.8 \mathrm{~Hz}, 1 \mathrm{H}, \mathrm{H}-4^{\prime}\right), 7.70\left(\mathrm{dd}, \mathrm{J}_{1}, \mathrm{~J}_{2}=7.2 \mathrm{~Hz}, 1 \mathrm{H}, \mathrm{H}-5^{\prime}\right), 4.52$ (s, 2H, H-4), $4.26\left(\mathrm{~s}, 2 \mathrm{H}, \mathrm{NH}_{2}\right) ;{ }^{13} \mathrm{C} \mathrm{NMR} \mathrm{(400} \mathrm{MHz,}$ DMSO-d $\left._{6}\right) \delta$ ppm 168.4, 153.4, 148.2, 136.9, 117.6, 114.5, 44.5; Anal. calcd for $\mathrm{C}_{7} \mathrm{H}_{9} \mathrm{ON}_{3}$ (151.16): C, 55.62; N, 27.80; H, 6.00. Found: C, 55.60; N, 27.78; H, 5.98\%.

\section{2-Amino-3-Phenyl-N-(Pyridin-2-yl)propanamide (S6b)}

Brown solid; yield: $85 \%$; m.p. $247{ }^{\circ} \mathrm{C}, \mathrm{R}_{\mathrm{f}}=0.27$ (pet.ether: ethyl acetate 3:1), FTIR ( $\left.v_{\max } \mathrm{cm}^{-1}\right) 3313$ (N-H, str.), 3268 ( $\mathrm{NH}_{2}$, str.), 1698 (C=O, amide, str.); ${ }^{1} \mathrm{H} \mathrm{NMR}$ (400 MHz, DMSO-d 6 ) $\delta$ ppm 9.85 (brs, $1 \mathrm{H}, \mathrm{NH}$ ), 8.63 (d, J=4.3 Hz, 1H, H-3'), 8.30 (d, J=8Hz, 1H, H-6'), 7.81 (t, J=7.7Hz,1H, H-4'), $7.70\left(\mathrm{dd}, \mathrm{J}_{1}, \mathrm{~J}_{2}=7.1 \mathrm{~Hz}, 1 \mathrm{H}, \mathrm{H}-5^{\prime}\right), 7.20-7.06(\mathrm{~m}, 5 \mathrm{H}, \mathrm{Ar}-\mathrm{H})$, 5.31 (d, J=4.8 Hz 1H, H-4), 4.28 (s, 2H, NH$), 3.69$ (m, 2H, $\mathrm{H}-5) ;{ }^{13} \mathrm{C}$ NMR (400 MHz, DMSO-d 6 ) $\delta$ ppm 170.6, 151.9, 147.4, 138.6, 136.4, 130.7, 129.8, 128.5, 127.4, 126.7, 119.2, 114.6, 57.6, 36.4; Anal. calcd for $\mathrm{C}_{14} \mathrm{H}_{15} \mathrm{ON}_{3}$ (241.28): $\mathrm{C}$, 69.69; N, 17.41; H, 6.27. Found: C, 69.58; N, 17.39; H, 6.20\%.

\section{2-Amino-4-Methyl-N-(Pyridine-2-yl)pentanamide (S6c)}

Brown solid; yield: $60 \%$; m.p. $231{ }^{\circ} \mathrm{C} ; \mathrm{R}_{\mathrm{f}}=0.35$ (pet.ether: ethyl acetate 3:1); FTIR ( $\left.v_{\max } \mathrm{cm}^{-1}\right) 3343$ (N-H, str.), 3269 ( $\mathrm{NH}_{2}$, str.), 1697 (C=O, amide, str.), ${ }^{1} \mathrm{H} \mathrm{NMR}$ (400 MHz, DMSO-d $_{6}$ ) $\delta$ ppm 9.68 (brs, $\left.1 \mathrm{H}, \mathrm{NH}\right), 8.65$ (d, J=4Hz, 1H,
H-3'), 8.29 (d, J=7.9Hz, 1H, H-6'), 7.92 (t, J=7.8Hz, 1H, H-4'), 7.79 (dd, $\left.\mathrm{J}_{1}, \mathrm{~J}_{2}=7 \mathrm{~Hz}, 1 \mathrm{H}, \mathrm{H}-5^{\prime}\right), 4.27\left(\mathrm{~s}, 2 \mathrm{H}, \mathrm{NH}_{2}\right), 5.11$ (t, $\mathrm{J}=5 \mathrm{~Hz}, 1 \mathrm{H}, \mathrm{H}-4), 2.01$ (m, 1H, H-6), 1.56 (dd, 2H, H-5), 1.0 $(\mathrm{d}, 6 \mathrm{H}, \mathrm{J}=6.4 \mathrm{~Hz}, \mathrm{H}-7 \mathrm{a}, 7 \mathrm{~b}) ;{ }^{13} \mathrm{C}$ NMR $\left(400 \mathrm{MHz}, \mathrm{DMSO}^{-\mathrm{d}_{6}}\right) \delta$ ppm 169.4, 153.8, 148.2, 137.6, 118.4, 115.7, 55.5, 43.7, 29.4, 25.3, 22.5; Anal. calcd for $\mathrm{C}_{11} \mathrm{H}_{17} \mathrm{ON}_{3}$ (207.27): $\mathrm{C}, 63.74 ; \mathrm{N}$, 20.27; H, 8.27. Found: C, 63.69; N, 20.25; H, 8.23\%.

\section{2-Amino-3-Methyl-N-(Pyridin-2-yl)pentanamide (S6d)}

White crystals; yield: $75 \%$; m.p. $236{ }^{\circ} \mathrm{C} ; \mathrm{R}_{\mathrm{f}}=0.35$ (pet.ether: ethyl acetate 3:1); FTIR ( $\left.v_{\max } \mathrm{cm}^{-1}\right) 3310$ (N-H, str.), 3270 $\left(\mathrm{NH}_{2}\right.$, str.), 1695 (C=O, amide, str.); ${ }^{1} \mathrm{H} \mathrm{NMR}$ (400 MHz, DMSO-d $\left.{ }_{6}\right) \delta$ ppm 9.96 (brs, $1 \mathrm{H}, \mathrm{NH}$ ), 9.49 (d, J=4Hz, 1H, H-3'), 8.32 (d, J=8Hz, 1H, H-6'), 8.20 (t, J=7.8Hz, 1H, H-4'), $7.90\left(\mathrm{dd}, \mathrm{J}_{1}, \mathrm{~J}_{2}=7.2 \mathrm{~Hz}, 1 \mathrm{H}, \mathrm{H}-5^{\prime}\right), 4.66$ (d, J=8Hz, 1H, H-4), 4.29 (s, 2H, $\mathrm{NH}_{2}$ ), 2.87 (m, 1H, H-5), 1.57 (m, 2H, H-6), 1.11 (d, $\mathrm{J}=8 \mathrm{~Hz}, 3 \mathrm{H}, \mathrm{H}-10), 0.91$ (t, J=8Hz, 3H, H-7); ${ }^{13} \mathrm{C} \mathrm{NMR}(400$ MHz, DMSO-d $\left.{ }_{6}\right) \delta$ ppm 170.4, 152.4, 149.8, 138.6, 118.5, 114.3, 58.5, 35.7, 26.4, 18.3, 12.2; Anal. calcd for $\mathrm{C}_{11} \mathrm{H}_{17} \mathrm{ON}_{3}$ (207.27): C, 63.74; N, 20.27; H, 8.27. Found: C, 63.70; N, 20.25; H, 8.23\%.

\section{In vitro Antioxidant Activity}

To preliminary evaluate the free radical scavenging potential of synthesized derivatives; an in vitro antioxidant activity assay was performed using DPPH. ${ }^{52}$ For the relative assay, a solution containing DPPH $(3 \mathrm{~mL})$ and methanol $(1 \mathrm{~mL})$ was designated as a negative control. Ascorbic acid was used as a reference standard. Different concentrations of test compounds were taken up to $3 \mathrm{~mL}$ and, $1 \mathrm{mM}$ DPPH solution in methanol was added. These mixtures were kept for 30 min under dark. UV spectrophotometer was used to assess the free radical scavenging potential of test compounds through measuring absorbance at $517 \mathrm{~nm}$. The inverse relationship between absorbance and test compound concentrations shows antioxidant potential. Furthermore, the color change also presents a potential indication that is from blue color to yellowish-orange color. Percent scavenging efficacy was determined using the formula:

$$
\begin{aligned}
& \% \text { radical } \\
& \text { scavenging }
\end{aligned}=\frac{\left(\begin{array}{l}
\text { absorbance } \\
\text { of control }
\end{array}-\begin{array}{l}
\text { absorbance } \\
\text { of test sample }
\end{array}\right)}{\text { absorbance of control }} \times 100
$$

\section{In vitro Anticancer Activity Preparation of Cells}

Human ovarian cancer cell lines A2780 and A2780CISR were purchased from ECACC (93112519, 93112517 for 
Table I The Concentration Range of Drugs is Prepared for Adding to Cells and the Final Concentration of Drugs After Addition to Cells

\begin{tabular}{|l|c|}
\hline Drugs & $\begin{array}{c}\text { Final Concentration of Drugs After Addition to } \\
\text { Cells }(\boldsymbol{\mu M})\end{array}$ \\
\hline Cisplatin & $0.16-20$ \\
S5b & $0.16-20$ \\
S6c & $0.16-20$ \\
S3b & $1.6-200$ \\
S3c & $1.6-200$ \\
S4c & $1.6-200$ \\
\hline
\end{tabular}

A2780 and A2780CISR respectively). The ovarian cancer cell lines A2780 (parent) and A2780CISR (cisplatinresistant) were grown in tissue culture flasks in an incubator at $37{ }^{\circ} \mathrm{C}$ in a humidified atmosphere of $5 \%$ $\mathrm{CO}_{2}$ and $95 \%$ air. 4500 to 5500 cells per well in $10 \%$ FCS/RPMI 1640 culture medium were seeded into flatbottomed 96-well culture plates and allowed to attach overnight. Cells were counted by Bio-Rad TC10 automated cell counter. $10 \mu \mathrm{L}$ of cell suspension was loaded onto the slide and inserted into the counter to count automatically. After counting cells, cell lines were seeded in two separate 96 well plates. $100 \mu \mathrm{L}$ of cell mixture (cells in the medium) were added to each well. Prepared drug concentrations are shown in Table 1. The plates were incubated for $24 \mathrm{~h}$ at $37{ }^{\circ} \mathrm{C}$ in a humidified atmosphere to allow the cells to attach.



Figure I Synthesis of 2-aminothiazole and 2-aminopyridine derivatives. 


\section{Drug Addition to Cells}

After the preparation of fivefold serial dilutions of the drugs in $10 \%$ FCS/RPMI 1640 medium, $100 \mu \mathrm{L}$ of each drug were added to equal volumes of cell culture in triplicate wells. The plates were then left to incubate in the carbon dioxide incubator $(5 \%$ carbon dioxide in the air, $\mathrm{pH}$ 7.4) for $72 \mathrm{~h}$ at $37^{\circ} \mathrm{C}$ in a humidified atmosphere.

\section{MTT Reduction Assay}

MTT [3-(4, 5-dimethylthiazol-2-yl)-2, 5-diphenyltetrazolium bromide] powder was dissolved in serum-freeRPMI-1640 medium at $1 \mathrm{mg} / \mathrm{mL}$ and filtered using DISMIC-25cs ADVANTEC cellulose acetate filter to remove any unwanted particles. The medium was removed and $50 \mu \mathrm{L}$ of the MTT solution was added to each well of 96-well plate and incubated for $4 \mathrm{~h}$ in the same carbon dioxide incubator. Cisplatin was used as a standard drug. After incubation, the MTT solution was removed and 150 $\mu \mathrm{L}$ of DMSO was added to each well. The living cells if still present remained attached at the bottom. The yellow formazan crystals produced from the reduction of MTT was dissolved in DMSO and the optical density (OD) of the resulting solution was read with the Bio-Rad, Microplate Reader, Benchmark at $570 \mathrm{~nm}$. The survival cell fractions were calculated according to the following equation of Mosmann (1983).

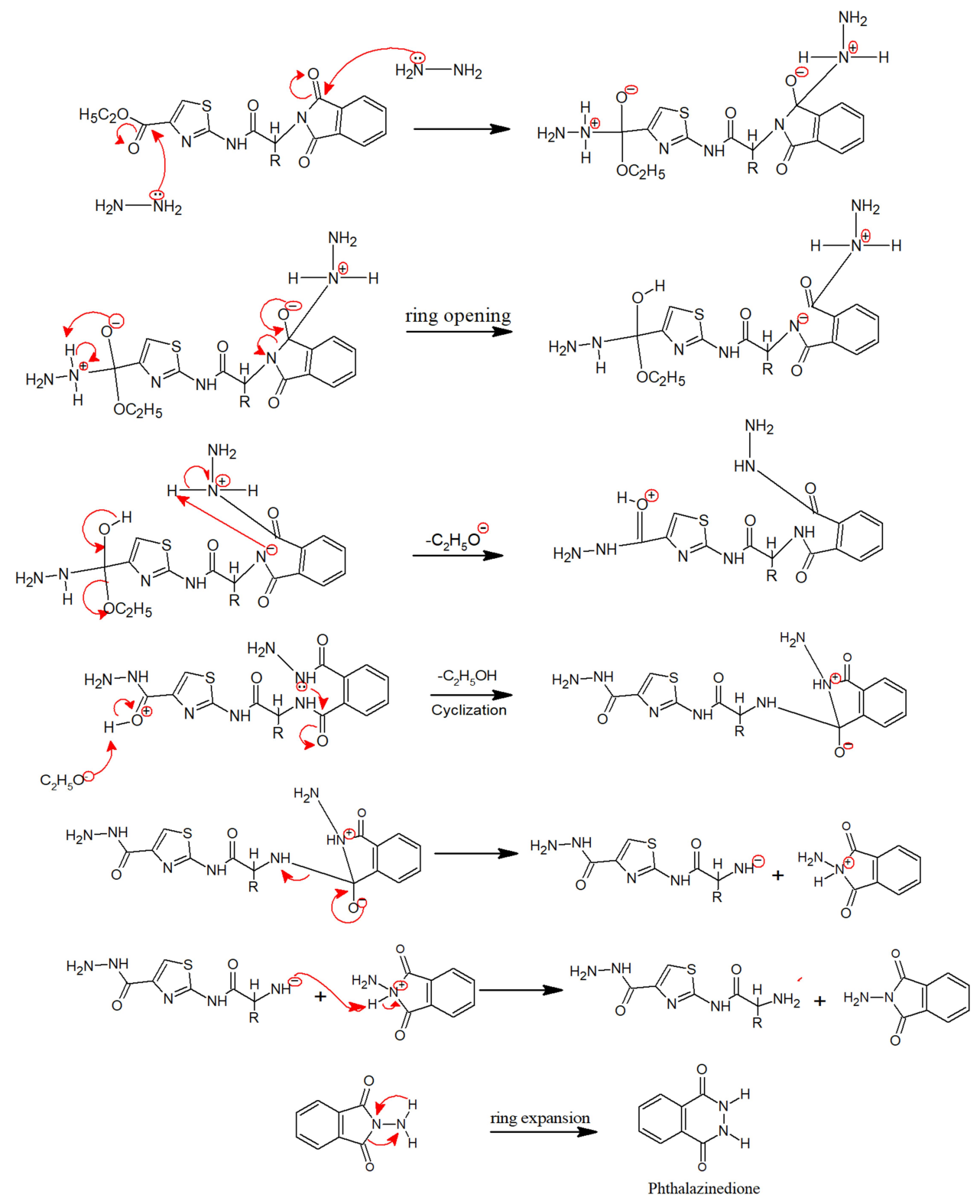

Figure 2 Proposed reaction mechanism to synthesize compound S5. 
$\begin{aligned} & \text { Survival } \\ & \text { cell fractions }\end{aligned}=\frac{\left(\begin{array}{l}\text { OD of } \\ \text { drug }\end{array}-\begin{array}{l}\text { Treated } \\ \text { cells }\end{array}-\begin{array}{l}\text { OD of } \\ \text { DMSO }\end{array}\right)}{(\text { OD of control }- \text { OD of DMSO })}$

Dosage response curves have been constructed by plotting the percentage of viable cells against drug concentration. The drug concentration required to cause $50 \%$ cell death $\left(\mathrm{IC}_{50}\right.$ value) was then calculated from the curve. For each drug concentration, at least three independent experiments were performed. ${ }^{53} \mathrm{RF}$ (Resistance factor) was also calculated using the following formula. ${ }^{46}$

$$
\text { Resistance factor }=\frac{\mathrm{IC}_{50} \text { in resistance cell line }}{\mathrm{IC}_{50} \text { in parent cell line }}
$$

\section{Molecular Docking}

Molecular docking studies of the compounds S3c, S5b and S6c with better in vitro anticancer activities were performed against eleven protein targets, selected from cell signaling pathways (ie, EGFR, VEGFR, PDGFR, $p^{110 a / P I 3 K, ~ R E T, ~ B C R-A B L, ~ c-K I T, ~}$ c-Raf, B-Raf, CTLA-4 and ALK) (PDB Codes: 1E90, 4LL0, 4AG8, 2L6W, 1ZZP, 2IVU, 3G0E, 3OMV, 2YJR, 3PPJ, 3OSK, respectively).

Structures of selected ligands ie S3c, S5b and S6c and standard inhibitors were prepared in ChemSketch and saved as mol files. Open Bable software was used to convert 2 dimensional (2D) structures to $3 \mathrm{D} .{ }^{54}$ Accelrys Discovery Studio Visualizer (version 4.1) was used to convert the structures in PDB file format for use in AutoDock Vina. ${ }^{55}$ The 3-dimensional crystal structures of cancer targets (proteins and DNA) were retrieved from the RCSB Protein Data Bank (http://www.rcsb.org/pdb). The proteins were set up for docking by removing attached ligand and crystallographic water molecules using Accelrys Discovery Studio Visualizer (version 4.1). The optimized proteins were then saved as a PDB file format for docking.

Molecular docking studies were performed using AutoDock Vina software (version 1.5.6) ${ }^{56}$ to determine binding affinities of ligands with respective protein targets and their binding energy values were also compared with anticancer standard drugs against respective targets. The AutoDock tool was used to add polar hydrogens in a protein molecule. The binding site for docking was designed such that the entire receptor molecule was included within the selection grid. Default parameters were used for docking. Binding affinities of synthesized and standard ligands were analyzed based on minimum binding energy values. Ligand protein binding interactions of complexes with minimum docking scores were analyzed using Accelrys Discovery Studio Visualizer (version 4.1).

\section{Statistical Analysis}

Graph Pad Prism 6.0 (Graph-Pad, San Diego, CA, United States) was used to analyze the data. For biochemical data, one way-ANOVA followed by Tukey's post hoc test was applied. Data were demonstrated as mean \pm SEM. Significant statistical differences were at $* \mathrm{p}<0.05$.

\section{Results}

\section{Chemistry}

New derivatives of Aminothiazole and aminopyridine derivatives were synthesized using the scheme given in Figure 1. Initially, the nitrogen group of amino acids was protected using phthalic anhydride S1(a-d). These were further reacted with thionyl chloride to yield corresponding acid chlorides and condensed with ethyl-2-aminothiazole-4-carboxylate and 2-aminopyridine to afford corresponding amide derivatives $\mathbf{S 3}(\mathbf{a}-\mathbf{d})$ and
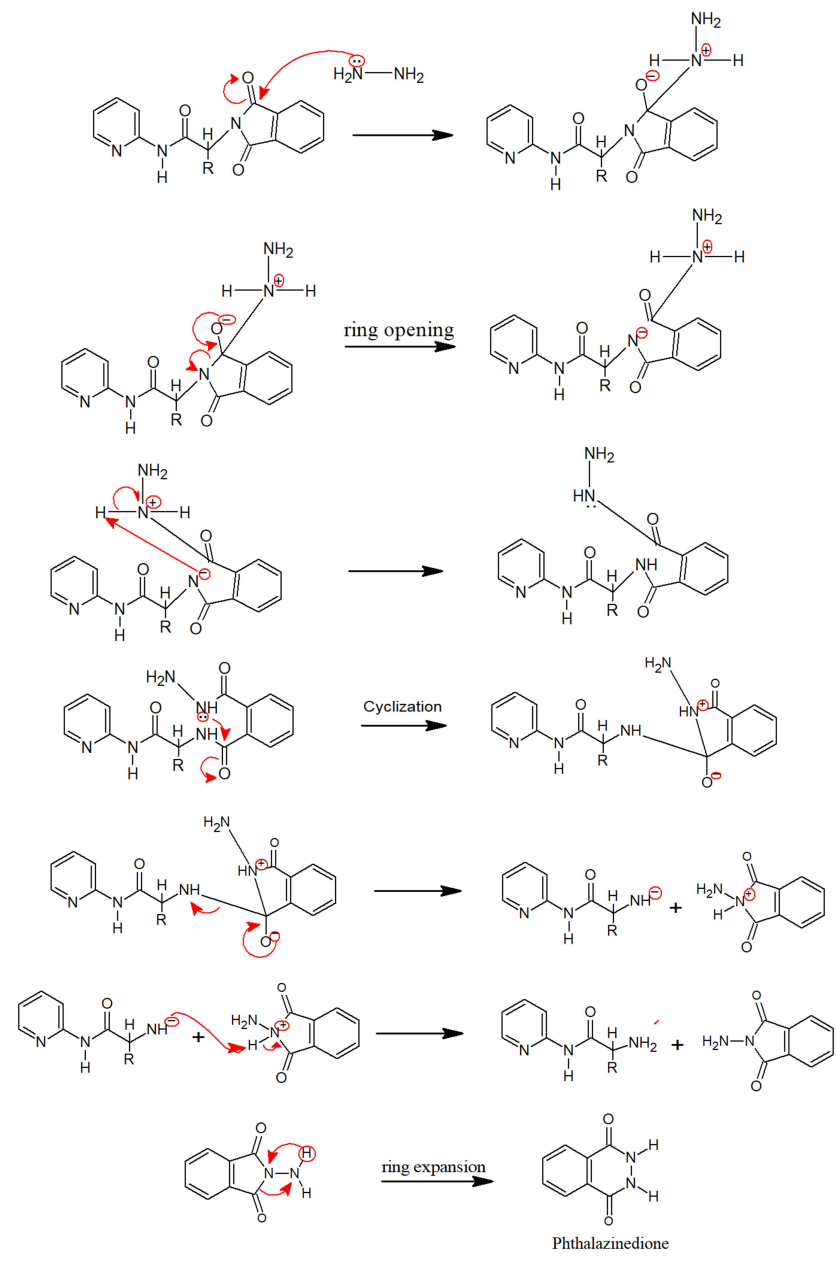

Figure 3 Proposed reaction mechanism to synthesize compound S6. 


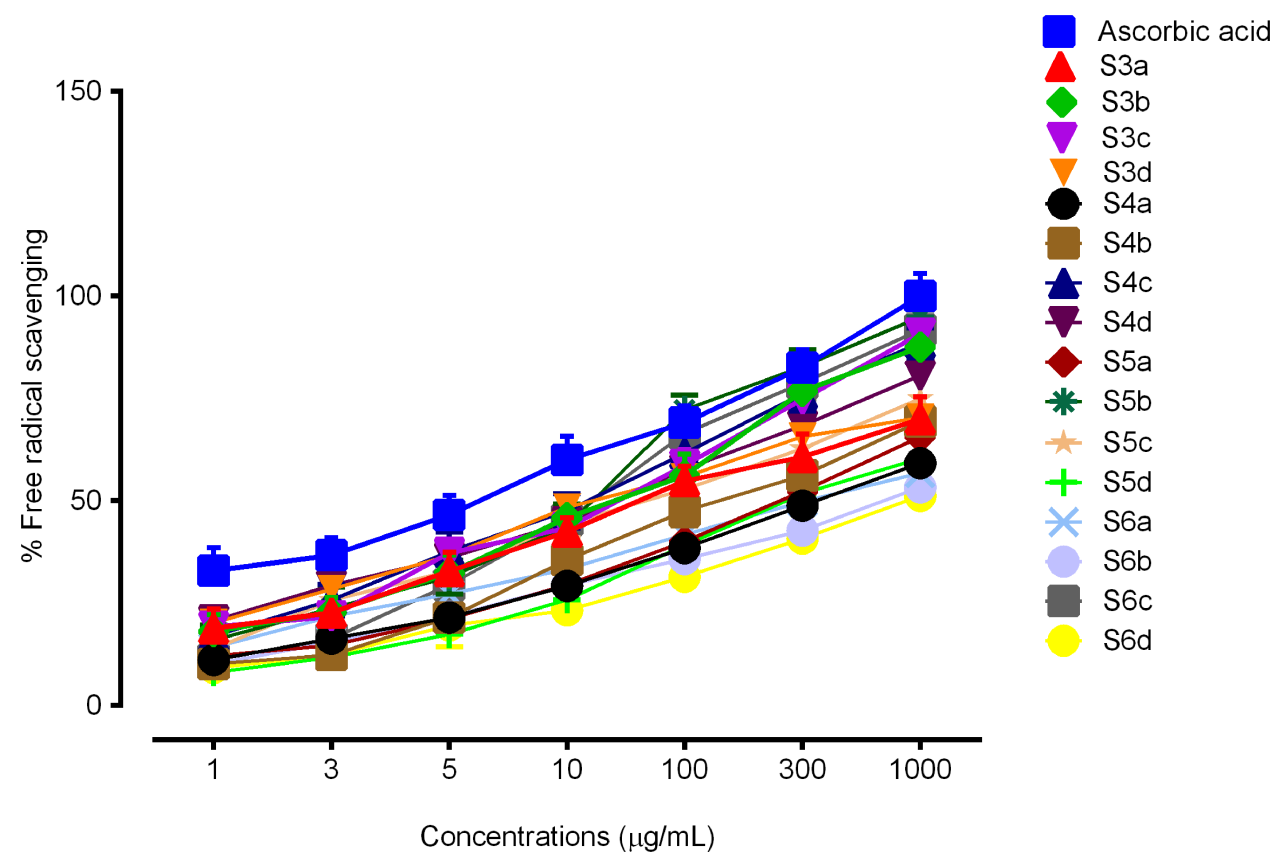

Figure 4 DPPH radical scavenging activity of synthesized derivatives.

S4(a-d) respectively. Finally, the N-deprotection of compounds S3(a-d) and S4(a-d) was carried out in the presence of hydrazine hydrate to yield compounds $\mathbf{S 5}(\mathbf{a}-\mathbf{d})$ and $\mathbf{S 6}(\mathbf{a}-\mathbf{d})$. The respective reaction mechanisms for $\mathrm{N}$-deprotection of compounds $\mathbf{S 3}$ and $\mathbf{S} 4$ are shown in Figures 2 and 3 respectively.

In the FTIR spectra, the presence of amide stretch in compounds S3(a-d) and S4(a-d) ranging 1698-1690 $\mathrm{cm}^{-1}$ confirmed the formation of an amide linkage. Further, the ${ }^{1} \mathrm{H}-\mathrm{NMR}$ data of synthesized analogues presented a singlet of -NH proton ranging $\delta=10.32-9.11 \mathrm{ppm}$, indicated the formation of coupled products. Similarly, in the case of S5(a-d) and S6(a-d) derivatives, the disappearance of carbonyl stretch for phthaloyl moiety in the range of $1635-1552 \mathrm{~cm}^{-1}$ confirmed the de-protection of amino acids.

\section{In vitro Antioxidant Activity}

2.2-diphenyl-1-picrylhydrazyl (DPPH) method was used to evaluate the antioxidant potential of newly synthesized compounds [S3(a-d), S4(a-d), S5(a-d), S6(a-d)] and the results are summarized in Figure 4. The antioxidant potential was calculated as the change in absorbance relative to the reference control. Probit regression was used to calculate $\mathrm{EC}_{50}$ values due to the non-linearity in regression. ${ }^{43}$ Compounds $\mathbf{S 3 b}, \mathbf{S 3 \mathbf { c }}$, S4c, S5b, and S6c showed significant activities with $\mathrm{EC}_{50}$ values, $16.25 \mu \mathrm{g} / \mathrm{mL}, 14.16 \mu \mathrm{g} / \mathrm{mL}, 17.03 \mu \mathrm{g} / \mathrm{mL}, 12.97 \mu \mathrm{g} /$ $\mathrm{mL}$, and $15.19 \mu \mathrm{g} / \mathrm{mL}$ respectively to the reference ascorbic acid which was found as $10.41 \mu \mathrm{g} / \mathrm{mL}$. Based on this preliminary assay, we selected five compounds (S3b, S3c, $\mathbf{S 4 c}, \mathbf{S 5 b}$, and S6c) for evaluation of their in vitro anticancer potential.

\section{In vitro Anticancer Activity}

Synthesized derivatives S3b, S3c, S4c, S5b, and S6c exhibited relative significant antioxidant activities were further evaluated for anticancer potential in parent and cisplatin-resistant ovarian cancer cell lines, A2780 and A2780CISR respectively, while cisplatin was used as a standard drug. $\mathrm{IC}_{50}$ and the resistance factor (RF) values of standard drug cisplatin and tested compounds are given in Table 2. All the tested compounds exhibited

Table 2 Anticancer Activity of the Synthesized Thiazole and Pyridine Derivatives Against A2780 (Parent) and A2780CISR (Cisplatin-Resistant) Ovarian Cancer Cell Lines, Expressed as $\mathrm{IC}_{50}$ Values and Compared to Cisplatin, the Standard Drug

\begin{tabular}{|l|c|c|c|}
\hline \multirow{2}{*}{ Tested Compounds } & \multicolumn{3}{|c|}{ IC $_{\mathbf{5 0}}(\boldsymbol{\mu M})$} \\
\cline { 2 - 4 } & $\mathbf{A 2 7 8 0} *$ & $\mathbf{A 2 7 8 0 \mathbf { C I S R } ^ { * * }}$ & $\mathbf{R F}^{\mathbf{a}}$ \\
\hline S3b & $38.56 \pm 1.98$ & $54.76 \pm 2.80$ & 1.42 \\
S3c & $15.57 \pm 2.37$ & $11.52 \pm 2.81$ & 0.74 \\
S4c & $39.14 \pm 1.79$ & $51.21 \pm 1.65$ & 1.31 \\
S5b & $25.57 \pm 2.83$ & $14.32 \pm 1.22$ & 0.56 \\
S6c & $31.45 \pm 0.02$ & $15.41 \pm 1.52$ & 0.49 \\
Cisplatin & $0.61 \pm 0.06$ & $16.43 \pm 1.45$ & 26.93 \\
\hline
\end{tabular}

Notes: *Parent ovarian cancer cell line. ${ }^{* *}$ Cisplatin resistant ovarian cancer cell line. ${ }^{a}$ Resistance factor. 


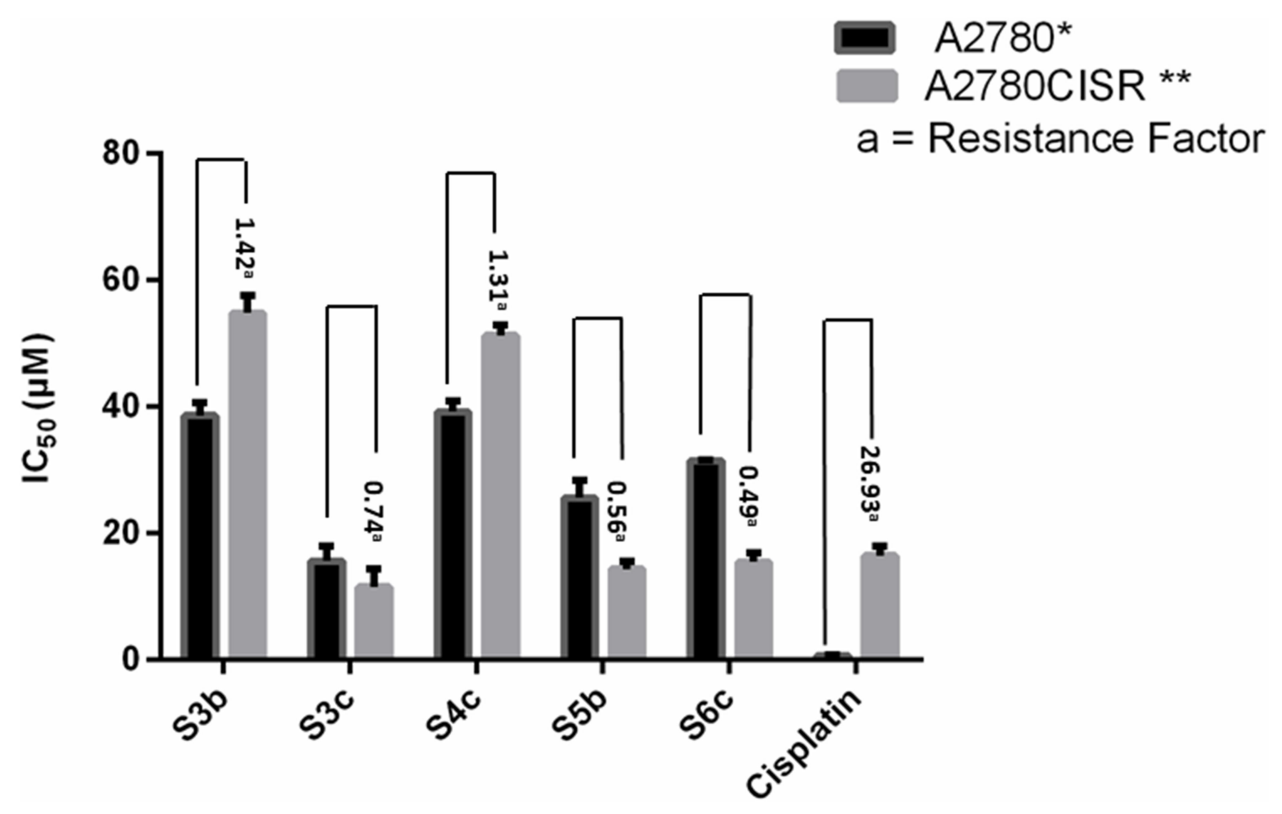

Figure $5 \mathrm{IC}_{50}$ values of compounds and cisplatin (standard) as observed in the parent (A2780*) and cisplatin-resistant (A2780CISR**) ovarian cancer cell lines. Data are presented as mean \pm SEM. One way ANOVA was used for the analysis of these results $(n=5)$.

a dose-dependent anti-proliferative effect against parent and cisplatin-resistant ovarian cancer cell lines. We demonstrated that compounds S3c, S5b, and S6c reversed the cisplatin resistance of cancer cells with $\mathrm{IC}_{50}$ values $11.52 \mu \mathrm{M}, 14.32 \mu \mathrm{M}$, and $15.41 \mu \mathrm{M}$ respectively relative to the standard drug cisplatin with $\mathrm{IC}_{50}$ values $16.43 \mu \mathrm{M}$. Furthermore, RF values for all the tested compounds S3b, S3c, S4c, S5b, and S6c were respectively ie $1.42,0.74,1.31,0.56$, and 0.49 , while for cisplatin was 26.93. Moreover, compounds S3c, S5b, and S6c exhibited more potency in cisplatin-resistant cell lines than the parent cells by displaying less $\mathrm{IC}_{50}$ values, associated with $\mathrm{RF}$ values of $0.74,0.56$, and 0.49 respectively. Furthermore, derivative S3c demonstrated inhibitory effects both in parent and cisplatinresistant ovarian cancer cell lines with an $\mathrm{IC}_{50}$ value of $15.57 \mu \mathrm{M}, 11.52 \mu \mathrm{M}$ respectively. This may be due to their variable effects on multiple proteins which are differentially expressed in sensitive and resistant cells (Figure 5).

Table 3 Binding Energies of Ligand Molecules (S3c, S5b and S6c) and Standard Drugs with Target Proteins

\begin{tabular}{|c|c|c|c|c|c|c|}
\hline \multirow[t]{2}{*}{ S.No. } & \multirow[t]{2}{*}{ Protein Targets } & \multirow[t]{2}{*}{ Standard Drugs } & Standard & S3c & S5b & S6c \\
\hline & & & \multicolumn{4}{|c|}{ Binding Energies (Kcal/mol) } \\
\hline I & $P^{110 a / P I 3 K}$ & Wortmannin & -9.9 & -9.0 & -8.5 & -7.9 \\
\hline 2 & EGFR & Cetuximab & mab* & -7.1 & -6.9 & -6.3 \\
\hline 3 & VEGFR & Sunitinib/Sorafenib & $-9.3 /-10.3$ & -6.7 & -8.6 & -6.9 \\
\hline 4 & PDGFR & Sunitinib/Sorafenib & $-5.5 /-6.7$ & -5.2 & -5.3 & -4.6 \\
\hline 5 & BCR-ABL & Imatinib & -9.3 & -5.3 & -5.6 & -5.8 \\
\hline 6 & RET & Vandetanib & -9.5 & -8.9 & -8.5 & -6.8 \\
\hline 7 & c-KIT & Sorafenib & -8.5 & -8.1 & -7.8 & -6.6 \\
\hline 8 & c-Raf & Sorafenib & -8.8 & -7.7 & -8.1 & -6.8 \\
\hline 9 & ALK & Crizotinib & -8.9 & -7.5 & -7 & -5.8 \\
\hline 10 & B-Raf & Sorafenib & -10.0 & -9 & -8.8 & -7.4 \\
\hline 11 & CTLA-4 & Ipilimumab & mab* & -5.5 & -5.7 & -5.0 \\
\hline
\end{tabular}

Note: *mab, Monoclonal antibody, structure not available. 

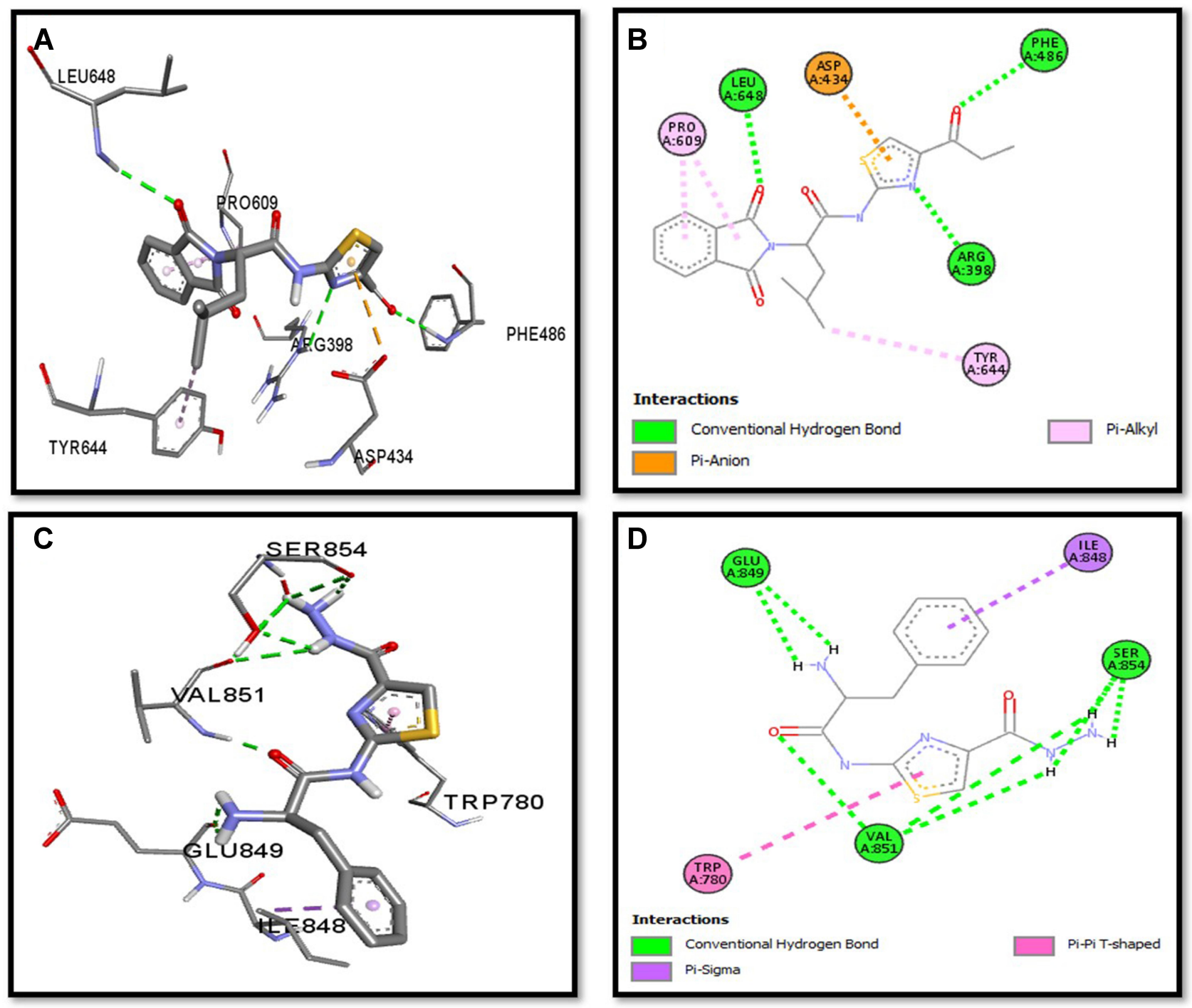

Figure 6 Post docking analysis visualized by Discovery Studio Visualizer in both 2D and 3D poses in the protein structures of $\mathrm{P}^{110 \mathrm{a}}$ isoform of PI3K. Interaction between S3C and $p^{110 a}$ isoform of PI3K (A and B), S5b and $p^{110 a}$ isoform of PI3K (C and D). 3D poses (A and $\left.\mathbf{C}\right)$ and 2D (B and $\left.\mathbf{D}\right)$.

\section{Docking Evaluation}

Synthesized compounds S3c, S5b, and S6c that exhibited significant in vitro anticancer activities were selected as ligands and docked against multiple protein targets such as EGFR, VEGFR, PDGFR, p p $^{110 a} /$ PI3K, RET, BCR-ABL, c-KIT, c-Raf, B-Raf, CTLA-4, and ALK. These targets were selected based upon their relative role in tumor development and are implicated in various cell signaling pathways. Further, the results of the docking study are summarized in Table 3. These results indicated that selected ligands demonstrated favorable interactions with multiple protein targets, which coincides with our experimental findings. Furthermore, compound S3c formed several hydrogen bonds with PDGFR, c-KIT, and ALK with the lowest binding energy values $-5.2 \mathrm{Kcal} / \mathrm{mol},-8.1 \mathrm{Kcal} / \mathrm{mol}$, and $-7.5 \mathrm{Kcal} / \mathrm{mol}$ respectively. Likely, compound $\mathbf{S 5 b}$ showed favorable docking against PDGFR, VEGFR, c-Raf, and CTLA-4 with binding energy values i.e $-5.3 \mathrm{Kcal} / \mathrm{mol},-8.6 \mathrm{Kcal} / \mathrm{mol},-8.1 \mathrm{Kcal} / \mathrm{mol},-5.7$ $\mathrm{Kcal} / \mathrm{mol}$ respectively that is comparable to standard inhibitors. It was analyzed that profound binding affinity was shown by compound S6c against BCR-Abl with the minimum free energy of binding $-5.8 \mathrm{Kcal} / \mathrm{mol}$. It was observed that these three derivatives possessed comparative binding affinity (possibly inhibitory) against EGFR and CTLA-4. As the $\mathrm{p}^{110 \mathrm{a}}$ isoform of PI3K is mainly involved in the development of ovarian cancer, all three 

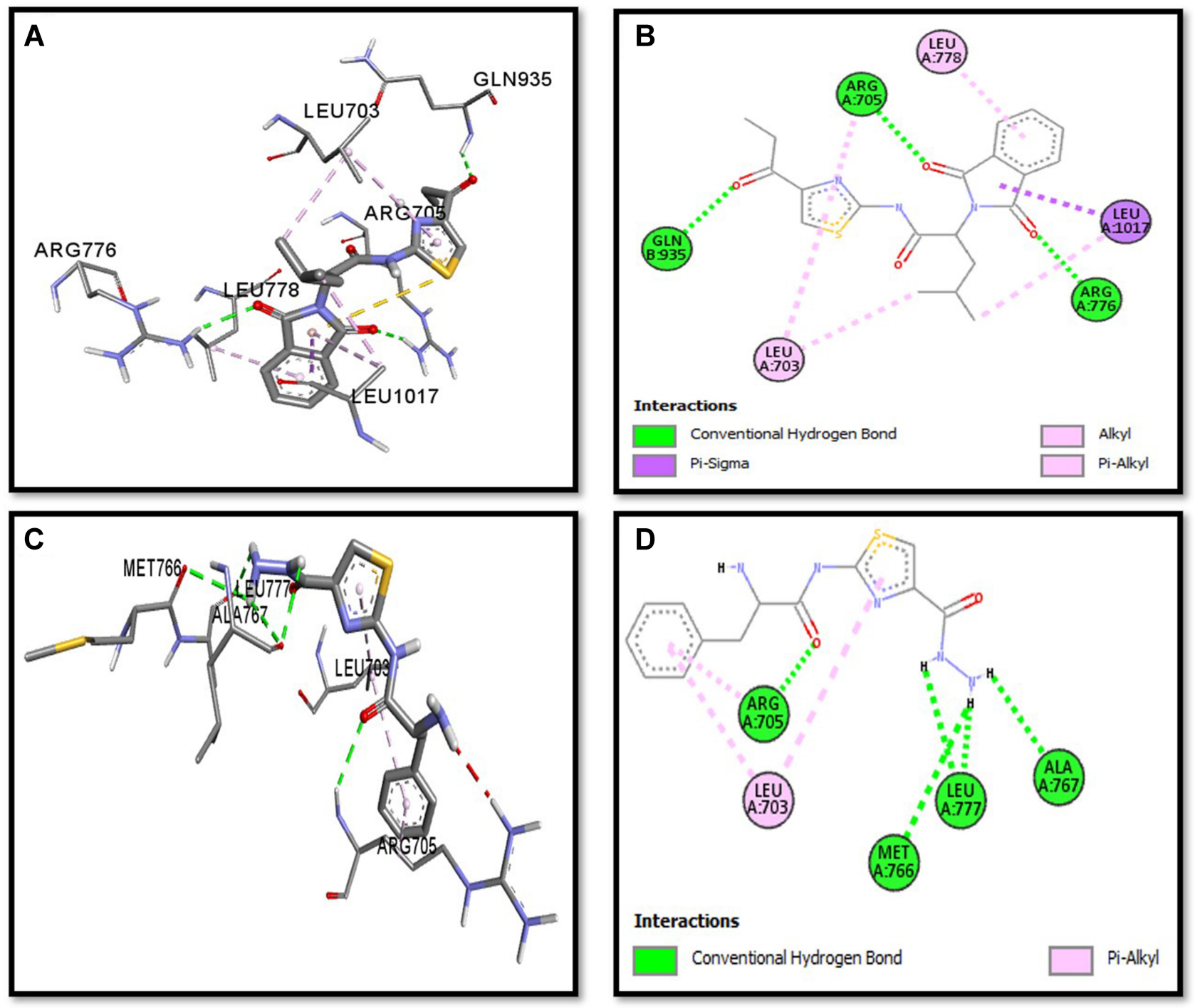

Figure 7 Post docking analysis visualized by Discovery Studio Visualizer in both 2D and 3D styles with EGFR. Interaction between S3c and EGFR (A and B), S5b and EGFR (C and D). 3D poses ( $\mathbf{A}$ and $\mathbf{C})$ and 2D (B and $\mathbf{D})$.

ligands were found to be active against this target. Compounds S3c, S5b, and S6c exhibited marked inhibition against $\mathrm{p}^{110 \mathrm{a}}$ isoform of PI3K with binding energy values of $-9.0 \mathrm{Kcal} / \mathrm{mol},-8.5 \mathrm{Kcal} / \mathrm{mol}$, and $-7.9 \mathrm{Kcal} /$ mol that is comparable to the reference compound ie $-9.9 \mathrm{Kcal} / \mathrm{mol}$.

Binding mode and 2D interactions of compounds S3c \& S5b with $\mathrm{p}^{110 \mathrm{a}}$ isoform of PI3K, EGFR, VEGFR, and PDGFR are expressed in Figures 6-9 respectively, demonstrating that these derivatives can significantly inhibit multiple targets in cellular pathways.

\section{Discussion}

In this study, we demonstrated the synthesis of new 2-aminothiazole and 2-aminopyridine derivatives, which possess amino acid moieties to execute antioxidant and anticancer potential. Interest in the chemotherapeutic activity of thiazoles was potentiated by the discovery of natural antineoplastic agents such as tiazofurin, bleomycin, netropsin, and thiazole netropsin. ${ }^{44}$ Moreover, many clinically useful anticancer drugs possess thiazole and pyridine as active moieties including dactinomycin and Imatinib mesylate respectively. ${ }^{29,35}$ 

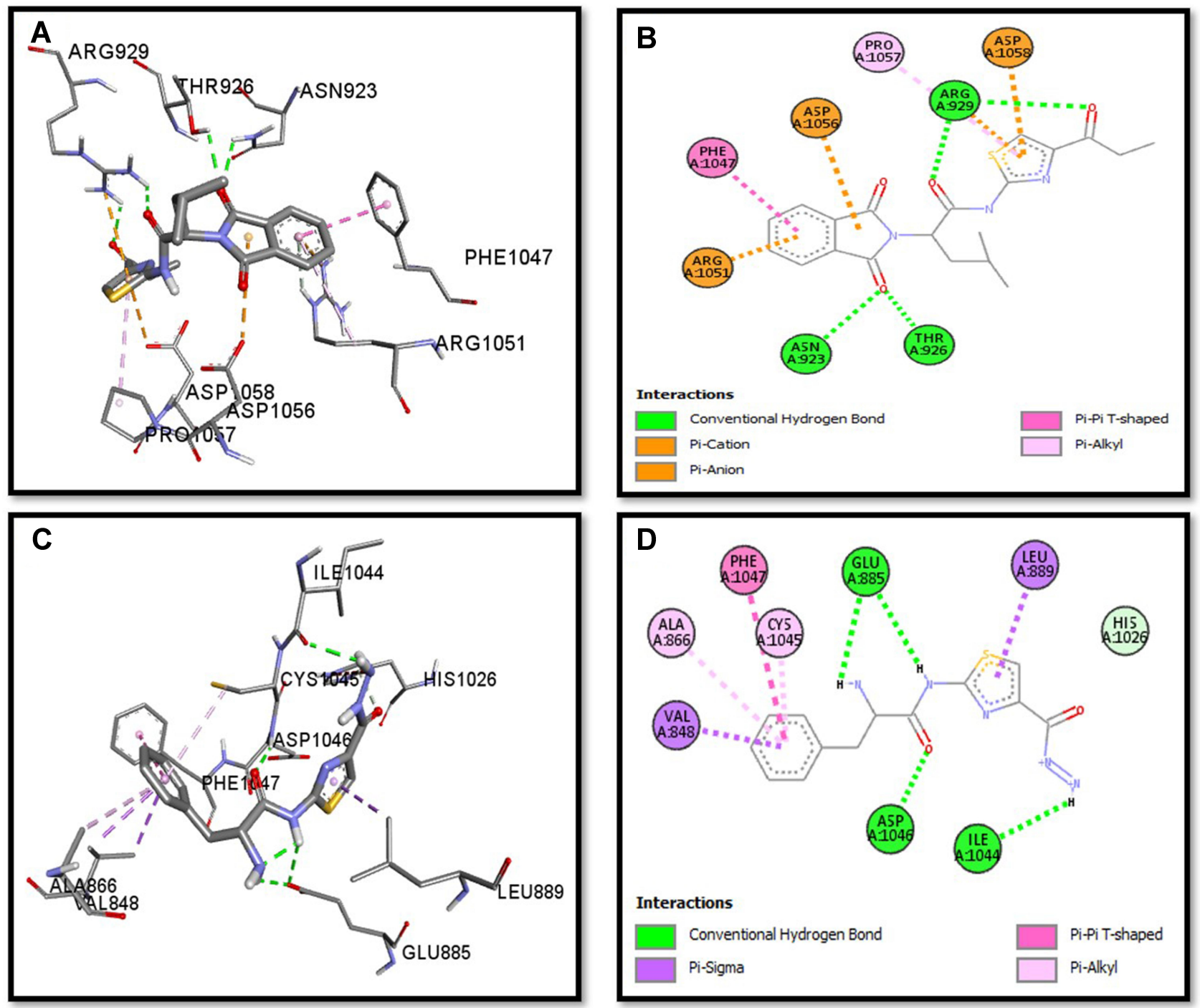

Figure 8 Post docking analysis visualized by Discovery Studio Visualizer in both 2D and 3D poses in the protein structures of VEGFR. Interaction between S3c and VEGFR (A and B), S5b and VEGFR (C and D). 3D poses (A and $\mathbf{C})$ and 2D (B and D).

The network-medicine approach implies a promising strategy by targeting several proteins in a network by rewiring network structures particularly proteins, as denatured or misfolded protein will induce vulnerability for chemoresistance and subsequent failure of cancer therapies. ${ }^{45}$ We demonstrated that our synthesized compounds can target multiple protein targets such as EGFR, VEGFR, PDGFR, p ${ }^{110 \mathrm{a}} / \mathrm{PI} 3 \mathrm{~K}, \mathrm{RET}$, BCR-ABL, c-KIT, c-Raf, B-Raf, CTLA-4, and ALK and thereby validating the concept of network-based approach. Derivative S3c showed an equivalent capacity of inhibition against PDGFR, c-KIT, and ALK target with lowest binding energy values $-5.2 \mathrm{Kcal} / \mathrm{mol},-8.1 \mathrm{Kcal} / \mathrm{mol}$, and -7.5 $\mathrm{Kcal} / \mathrm{mol}$ respectively. Moreover, compound $\mathbf{S 5 b}$ exhibited strong bindings against cancer targets PDGFR, VEGFR, c-Raf, and CTLA-4 with energy values of -5.3 $\mathrm{Kcal} / \mathrm{mol},-8.6 \mathrm{Kcal} / \mathrm{mol},-8.1 \mathrm{Kcal} / \mathrm{mol},-5.7 \mathrm{Kcal} / \mathrm{mol}$ respectively. The $\mathrm{p}^{110 \mathrm{a}}$ isoform of PI3K is the main target involved in the development of ovarian cancer, ${ }^{8}$ and all three ligands (S3c, S5b, and S6c) displayed a good binding affinity with this target with binding energy values of $-9.0 \mathrm{Kcal} / \mathrm{mol}, \quad-8.5 \mathrm{Kcal} / \mathrm{mol}$ and $-7.9 \mathrm{Kcal} / \mathrm{mol}$ respectively.

Redox imbalance and oxidative stress are hallmarks of cancers, so, antioxidants play a significant role in cancer treatment by ameliorating levels of free radicals and oxidative stress. ${ }^{37,38}$ Therefore, the antioxidant activity of synthesized compounds was performed to 

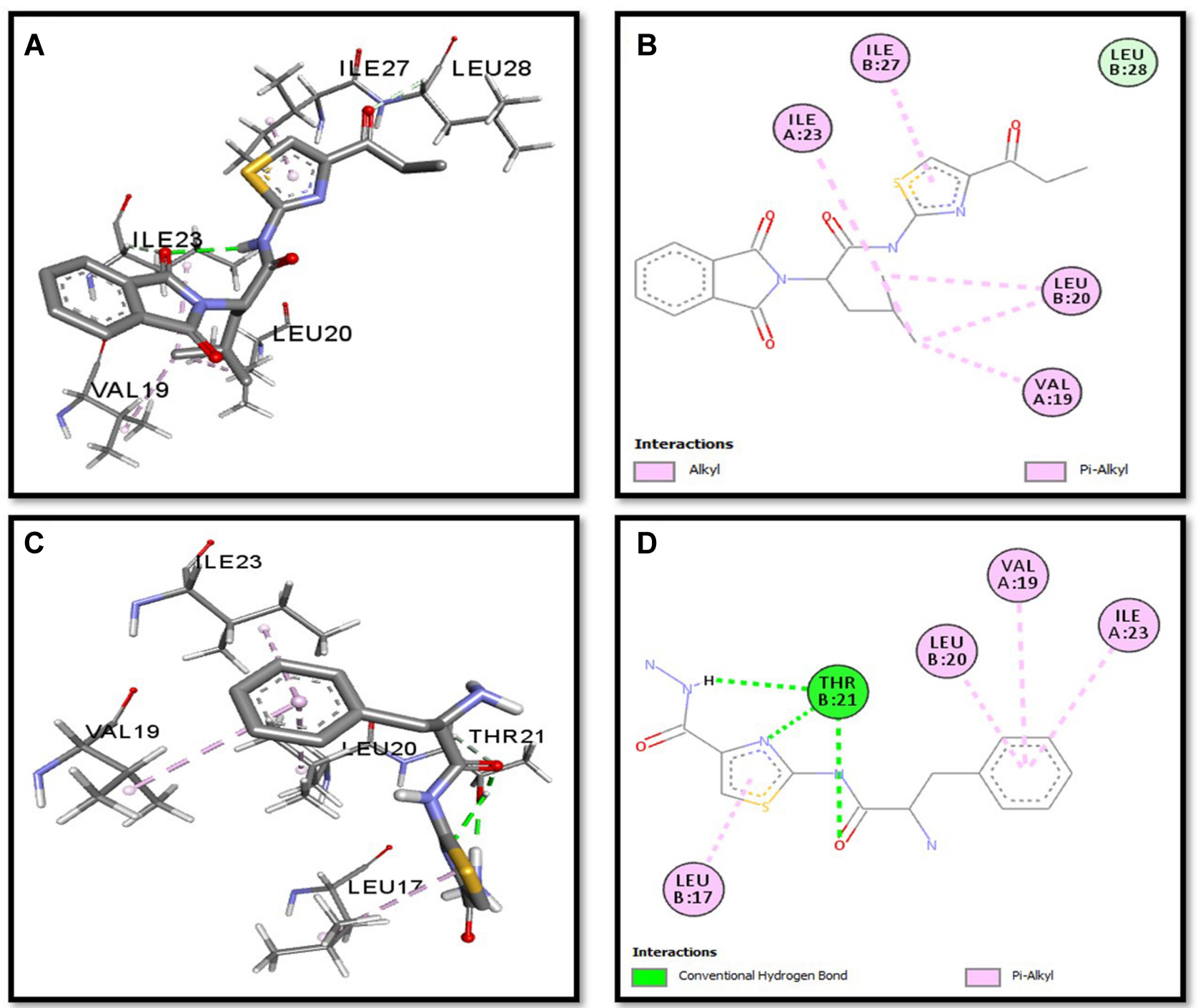

Figure 9 Post docking analysis visualized by Discovery Studio Visualizer in both 2D and 3D styles with PDGFR. Interaction between S3c and PDGFR (A and B), S5b and PDGFR (C and D). 3D poses (A and $\mathbf{C})$ and 2D (B and D).

preliminary screen the free radical scavenging potential. Based on our results, five compounds $\mathbf{S 3 b}, \mathbf{S 3 c}$, S4c, S5b, and S6c with an $\mathrm{EC}_{50}$ value of $16.25 \mathrm{ug} / \mathrm{mL}$, $14.16 \mathrm{ug} / \mathrm{mL}, 17.03 \mathrm{ug} / \mathrm{mL}, 12.97 \mathrm{ug} / \mathrm{mL}$, and 15.19 $\mathrm{ug} / \mathrm{mL}$ respectively were selected for evaluation of the in vitro anticancer potential in comparison to ascorbic acid.

Cisplatin is clinically used in a wide spectrum of cancers, however, its therapeutic implications are limited by its toxicities, and tumor resistance. ${ }^{9}$ To overcome, we incorporated amino acids conjugation into thiazole and pyridine scaffold to minimize toxicities by enhancing selective cellular permeability of the heterocyclic scaffold. ${ }^{41}$ Cellular mechanisms responsible for cisplatin resistance are multifactorial that restrict their clinical use. ${ }^{12,13}$ Our results revealed that compounds S3c, S5b, and S6c were found to be more active in cisplatin-resistant cell lines than the parent cells by displaying less $\mathrm{IC}_{50}$ values, $11.52 \mu \mathrm{M}$, $14.32 \mu \mathrm{M}$, and $15.41 \mu \mathrm{M}$ respectively relative to the standard cisplatin which was $16.43 \mu \mathrm{M}$ along with stable RF values of $0.74,0.56$, and 0.49 respectively. RF determines the ability of a compound to induce cell death in resistant cells and RF value less than 2 is an indication of significant activity of a compound against resistant cell lines. ${ }^{46}$ The RF for all the tested compounds S3b, S3c, S4c, S5b, and S6c were noteworthy ie $1.42,0.74,1.31,0.56$, and 0.49 respectively, while, for cisplatin is 26.93 . This may be due to their variable effects on genes and proteins which are 
differentially expressed in sensitive and resistant cells such as oncogenes (c-fos, c-myc, H-ras, c-jun, and $c-a b l)$, tumor suppressor genes ( $p 53)$, transcription factors, and regulatory proteins involved in signal transduction $_{\text {pathways. }}{ }^{14}$ Moreover, compound S3c demonstrated equal effectiveness in both parent and cisplatin-resistant ovarian cancer cells with an $\mathrm{IC}_{50}$ value of $15.57 \mu \mathrm{M}, 11.52 \mu \mathrm{M}$ respectively.

Therefore, it can be inferred that compounds S3c, S5b, and S6c can be considered as potential leads or hits for the development of new anticancer agents for cisplatin-resistant ovarian cancer. More detailed studies are required to delineate the detailed underlying mechanism of these compounds in different cancer cell lines and targets.

\section{Conclusion}

In conclusion, our findings demonstrated that derivatives S3c, S5b and S6c were found to be more active in cisplatin-resistant ovarian cancer cell lines than cisplatin-sensitive cells. Therefore, these compounds may be used as potential anticancer lead candidates for further screening because they might reduce oxidative stress in cancerous cells and also possess multiple target inhibitory potential.

\section{Author Contributions}

All authors made substantial contributions to conception and design, acquisition of data, or analysis and interpretation of data; took part in drafting the article or revising it critically for important intellectual content; agreed to submit to the current journal; gave final approval of the version to be published, and agree to be accountable for all aspects of the work.

\section{Funding}

This work was supported by Shenzhen-Hong Kong Institute of Brain Science-Shenzhen Fundamental Research Institutions No: 2019SHIBS0004, Shenzhen, 518055, China.

\section{Disclosure}

The authors report no conflicts of interest for this work.

\section{References}

1. Ghoneum A, Said N. PI3K-AKT-mTOR and NFкB pathways in ovarian cancer: implications for targeted therapeutics. Cancers. 2019;11: 949. doi:10.3390/cancers 11070949
2. Wu YH, Huang YF, Chen CC, Huang CY, Chou CY. Comparing PI3K/Akt inhibitors used in ovarian cancer treatment. Front Pharmacol. 2020;11:206. doi:10.3389/fphar.2020.00206

3. Lee MJ, Ye AS, Gardino AK, et al. Sequential application of anticancer drugs enhances cell death by rewiring apoptotic signaling networks. Cell. 2012;149(4):780-794. doi:10.1016/j.cell.2012.03.031

4. Zhang F, Ren C, Lau KT. A network medicine approach to build a comprehensive atlas for the prognosis of human cancer. Brief Bioinform. 2016;17(6):1044-1059. doi:10.1093/bib/bbw076

5. Liu X, Wu J, Zhang D, et al. Network pharmacology-based approach to investigate the mechanisms of Hedyotis diffusa willd. in the treatment of gastric cancer. Evid Based Complement Alternat Med. 2018. doi:10.1155/2018/7802639

6. Nasser AA, Eissa IH, Oun MR. Discovery of new pyrimidine-5-carbonitrile derivatives as anticancer agents targeting $\mathrm{EGFR}^{\mathrm{WT}}$ and $\mathrm{EGFR}^{\mathrm{T} 790 \mathrm{M}}$. Org Biomol Chem. 2020;18 (38):7608-7634. doi:10.1039/D0OB01557A

7. Pawson T, Linding R. Network medicine. FEBS Lett. 2008;58 2:1266-1270. doi:10.1016/j.febslet.2008.02.011

8. Liu R, Chen Y, Liu G, et al. PI3K/AKT pathway as a key link modulates the multidrug resistance of cancers. Cell Death Dis. 2020;11:797. doi:10.1038/s41419-020-02998-6

9. Amable L. Cisplatin resistance and opportunities for precision medicine. Pharmacol Res. 2016;106:27-36. doi:10.1016/j.phrs.2016.01.001

10. Navya PN, Kaphle A, Daima HK. Nanomedicine in sensing, delivery, imaging and tissue engineering: advances, opportunities and challenges. Nanoscience. 2019;5:30-56.

11. Navya PN, Kaphle A, Srinivas SP, Bhargava SK, Rotello VM, Daima HK. Current trends and challenges in cancer management and therapy using designer nanomaterials. Nano Converge. 2019;6:23.

12. Chen S, Chang J. New insights into mechanisms of cisplatin resistance: from tumor cell to microenvironment. Int J Mol Sci. 2019;20 (17):4136. doi:10.3390/ijms20174136

13. Ferreira JA, Peixoto A, Neves M, et al. Mechanisms of cisplatin resistance and targeting of cancer stem cells: adding glycosylation to the equation. Drug Resist. 2016;24:34-54.

14. Zhou J, Kang Y, Chen L, et al. The drug-resistance mechanisms of five platinum-based antitumor agents. Front Pharmacol. 2020;11:343.

15. Vitaku E, Smith DT, Njardarson JT. Analysis of the structural diversity, substitution patterns, and frequency of nitrogen heterocycles among U.S. FDA approved pharmaceuticals. J Med Chem. 2014;57:10257-10274. doi:10.1021/jm501100b

16. Bosenbecker J, Bareno VDO, Difabio R, et al. Synthesis and antioxidant activity of 3-(pyridin-2-yl,ethyl)-1,3-thiazinan(thiazolidin)-4-ones. J Biochem Mol Toxic. 2014;28:425-432. doi:10.1002/jbt.21581

17. Sraa AM, Mastoura ME, Heba HS, et al. Synthesis and biological evaluation of some novel thiazole-based heterocycles as potential anticancer and antimicrobial agents. Molecules. 2019;24:539. doi:10.3390/molecules24030539

18. Hussein AM, Khames AA, El-Adasy AA, et al. Design, synthesis and biological evaluation of new 2-aminothiazole scaffolds as phosphodiesterase type 5 regulators and COX-1/COX-2 inhibitors. RSC Adv. 2020;10(50):29723-29736. doi:10.1039/D0RA05561A

19. Rodríguez-Rangel S, Bravin AD, Ramos-Torres KM, Brugarolas P, Sánchez-Rodríguez JE. Structure-activity relationship studies of four novel 4-aminopyridine $\mathrm{K}^{+}$channel blockers. Sci Rep. 2020;10(1):52. doi:10.1038/s41598-019-56245-w

20. Hersi F, Omar HA, Al-Qawasmeh RA, et al. Design and synthesis of new energy restriction mimetic agents: potent anti-tumor activities of hybrid motifs of aminothiazoles and coumarins. Sci Rep. 2020;10 (1):2893. doi:10.1038/s41598-020-59685-x

21. El-Naggar AM, Eissa IH, Belal A, El-Sayed AA. Design, eco-friendly synthesis, molecular modeling and anticancer evaluation of thiazol-5(4H)-ones as potential tubulin polymerization inhibitors targeting the colchicine binding site. RSC Adv. 2020;10 (5):2791-2811. doi:10.1039/C9RA10094F 
22. Eissa IH, Metwaly AM, Belal A, et al. Discovery and antiproliferative evaluation of new quinoxalines as potential DNA intercalators and topoisomerase II inhibitors. Arch Pharm. 2019;352(11): e1900123. doi:10.1002/ardp.201900123

23. El-Helby AA, Sakr H, Eissa IH, Al-Karmalawy AA, El-Adl K. Benzoxazole/benzothiazole-derived VEGFR-2 inhibitors: design, synthesis, molecular docking, and anticancer evaluations. Arch Pharm. 2019;352(12):e1900178. doi:10.1002/ardp.201900178

24. Abu-Melha S, Edrees MM, Riyadh SM, Abdelaziz MR, Elfiky AA, Gomha SM. Clean grinding technique: a facile synthesis and in silico antiviral activity of hydrazones, pyrazoles, and pyrazines bearing thiazole moiety against SARS-CoV-2 main protease (Mro $)$. Molecules. 2020;25(19):4565. doi:10.3390/ molecules25194565

25. Sayed AR, Gomha SM, Taher EA, et al. One-pot synthesis of novel thiazoles as potential anti-cancer agents. Drug Des Devel Ther. 2020;14:1363-1375. doi:10.2147/DDDT.S221263

26. Abdelrazek FM, Gomha SM, Shaaban MEB, et al. One-pot three-component synthesis and molecular docking of some novel 2-thiazolyl pyridines as potent antimicrobial agents. Mini Rev Med Chem. 2019;19(6):527-538. doi:10.2174/1389557518666181019124104

27. Abdelrazek FM, Gomha SM, Abdelrahman AH, Metz P, Sayed MA. A facile synthesis and drug design of some new heterocyclic compounds incorporating pyridine moiety and their antimicrobial evaluation. Lett Drug Des Discov. 2017;14(7):752-762. doi:10.21 74/1570180814666161128120240

28. Ali AR, El-Bendary ER, Ghaly MA, et al. Novel acetamidothiazole derivatives: synthesis and in vitro anticancer evaluation. Eur J Med Chem. 2013;69:908-919. doi:10.1016/j.ejmech.2013.08.021

29. Popsavin M, Spaic S, Svircev M, et al. Synthesis and antitumour activity of new tiazofurin analogs bearing a 2,3-anhydro functionality in the furanose ring. Bioorg Med Chem Lett. 2007;17:4123-4127. doi:10.1016/j.bmcl.2007.05.050

30. Schiavi B, Ahond A, Al-Mourabit A, et al. Synthesis of 5-deazathiogirollines: analogs of a natural anticancer agent. Tetrahedron. 2002;58:4201-4215. doi:10.1016/S0040-4020(02)00383-6

31. Yeh CB, Su CJ, Hwang JM, et al. Therapeutic effects of cantharidin analogues without bridging ether oxygen on human hepatocellular carcinoma cells. Eur J Med Chem. 2010;45:3981-3985. doi:10.1016/ j.ejmech.2010.05.053

32. Abbas I, Gomha S, Elaasser M, Bauomi M. Synthesis and biological evaluation of new pyridines containing imidazole moiety as antimicrobial and anticancer agents. Turk J Chem. 2015;39(2):334-346. doi:10.3906/kim-1410-25

33. Gomha SM, Muhammad ZA, Abdel-aziz HM, Matar IK, ElSayed AA. Green synthesis, molecular docking and anticancer activity of novel 1,4-dihydropyridine-3,5-Dicarbohydrazones under grind-stone chemistry. Green Chem Lett Rev. 2020;13(1):6-17. doi:10.1080/17518253.2019.1710268

34. Gomha SM, Abdelrazek FM, Abdelrahman AH, Metz P. Synthesis of some new pyridine-based heterocyclic compounds with anticipated antitumor activity. J Heterocycl Chem. 2018;55(7):1729-1737. doi: $10.1002 /$ jhet. 3210

35. El-Naggar M, Almahli H, Ibrahim HS, Eldehna WM, AbdelAziz HA. Pyridine-ureas as potential anticancer agents: synthesis and in vitro biological evaluation. Molecules. 2018;23(6):1459. doi:10.3390/molecules23061459

36. Klaunig JE. Oxidative stress and cancer. Curr Pharm Des. 2018;24 (40):4771-4778. doi:10.2174/1381612825666190215121712

37. Hayes JD, Dinkova-Kostova AT, Tew KD. Oxidative stress in cancer. Cancer Cell. 2020;38(2):167-197.

38. Kruk J, Aboul-Enein HY. Reactive oxygen and nitrogen species in carcinogenesis: implications of oxidative stress on the progression and development of several cancer types. Mini Rev Med Chem. 2017;17(11):904-919. doi:10.2174/1389557517666170228115324
39. Alizadeh SR, Hashemi SM. Development and therapeutic potential of 2-aminothiazole derivatives in anticancer drug discovery. Med Chem Res. 2021;1-36. doi:10.1007/s00044-020-02686-2

40. Gomha SM, Muhammad ZA, Abdel-aziz MR, Abdel-aziz HM, Gaber HM, Elaassere MM. One-pot synthesis of new thiadiazolyl-pyridines as anticancer and antioxidant agents. J Heterocycl Chem. 2018;55(2):530-536. doi:10.1002/jhet.3088

41. Arun R, Ashok KCK. Synthesis, hydrolysis and pharmacodynamics profiles of novel prodrugs of mefenamic acid. Int J Curr Pharm Res. 2009;1:47-55.

42. Prakasha KC, Raghavendra GM, Harisha R, et al. Design, synthesis and antimicrobial screening of amino acids conjugated 2-amino4-arylthiazole derivatives. Int J Pharm Pharm Sci. 2011;3:120-125.

43. Locatelli M, Gindro R, Travaglia F, et al. Study of DPPH-scavenging activity: development of a free software for the correct interpretation of data. Food Chem. 2009;114:889-897. doi:10.1016/j.foodchem. 2008.10.035

44. Al-Saadi SM, Hassan MF, Sherif AFR. Synthesis and biological evaluation of some 2,4,5- trisubstituted thiazole derivatives as potential antimicrobial and anticancer agents. Arch Pharm Chem Life Sci. 2008;341:424-434. doi:10.1002/ardp.200800026

45. Barabasi AL, Gulbahce N, Loscalzo J. Network medicine: a network-based approach to human disease. Nat Rev Genet. 2011;12:56-68. doi:10.1038/nrg2918

46. Laila A, Philip B, Charles C, et al. Synergism from combinations of tris(benzimidazole) monochloroplatinum(II) chloride with capsaicin, quercetin, curcumin and cisplatin in human ovarian cancer cell lines. Anticancer Res. 2014;34:5453-5464.

47. Madhu G, Nanjaian M, Darpan K. Facile synthesis and characterization of 2-aminothiazole-4-carbohydrazide. Int J Rec Adv Pharm Res. 2011;2:27-29.

48. Hassan M, Farzana S, Uzma Y, et al. Preparation of optically active amino acid derivatives of some methylated 5-amino azaheterocycles. Turk J Chem. 2000;24:165-175.

49. Cyril O, Didier ML, Johan W, et al. Synthesis and anticonvulsant activity of $N, N$-phthaloyl derivatives of central nervous system inhibitory amino acids. Arch Pharm Pharm Med Chem. 2001;334:323-331. doi:10.1002/1521-4184(200110)334:10<323:: AID-ARDP323>3.0.CO;2-O

50. Abhilasha V, Nirupam D, Meenakshi D, et al. Conjugation of some NSAIDs with 5-phenyl-2-aminothiazole for reduced ulcerogenicity. Thai J Pharm Sci. 2010;34:49-57.

51. Sinead OMC, Joan EM, Stanley RM, et al. Intermediates in the Ing-Manske reaction. ARKIVOC. 2003;VII:180-189.

52. Molyneux $P$. The use of the stable free radical diphenyl picryl hydrazyl (DPPH) for estimating antioxidant activity. Songklanakarin $\mathrm{J} \mathrm{Sci}$ Technol. 2004;26:211-219.

53. Mosmann T. Rapid colorimetric assay for cellular growth and survival: application to proliferation and cytotoxicity assays. J Immunol Methods. 1983;65:55-63. doi:10.1016/0022-1759(83)90303-4

54. O'Boyle NM, Banck M, James CA, et al. Open Babel: an open chemical toolbox. J Cheminform. 2011;3:1-14. doi:10.1186/17582946-3-1

55. Rao SN, Head MS, Kulkarni A, et al. Validation studies of the site-directed docking program LibDock. J Chem Inf Model. 2007;47:2159-2171. doi:10.1021/ci6004299

56. Morris GM, Goodsell DS, Halliday RS, et al. Automated docking using a lamarckian genetic algorithm and and empirical binding free energy function. J Comput Chem. 1998;19:1539-1662. doi:10.1002/ (SICI)1096-987X(19981115)19:14<1639::AID-JCC10>3.0.CO;2-B 


\section{Publish your work in this journal}

Drug Design, Development and Therapy is an international, peerreviewed open-access journal that spans the spectrum of drug design and development through to clinical applications. Clinical outcomes, patient safety, and programs for the development and effective, safe, and sustained use of medicines are a feature of the journal, which has also been accepted for indexing on PubMed Central. The manuscript management system is completely online and includes a very quick and fair peer-review system, which is all easy to use. Visit http://www. dovepress.com/testimonials.php to read real quotes from published authors. 